\title{
ON NONLINEAR DELAY DIFFERENTIAL EQUATIONS
}

\author{
A. ISERLES
}

\begin{abstract}
We examine qualitative behaviour of delay differential equations of the form$$
y^{\prime}(t)=h(y(t), y(q t)), \quad y(0)=y_{0},
$$

where $h$ is a given function and $q>0$. We commence by investigating existence of periodic solutions in the case of $h(u, v)=f(u)+p(v)$, where $f$ is an analytic function and $p$ a polynomial. In that case we prove that, unless $q$ is a rational number of a fairly simple form, no nonconstant periodic solutions exist. In particular, in the special case when $f$ is a linear function, we rule out periodicity except for the case when $q=1 / \operatorname{deg} p$. If, in addition, $p$ is a quadratic or a quartic, we show that this result is the best possible and that a nonconstant periodic solution exists for $q=\frac{1}{2}$ or $\frac{1}{4}$, respectively. Provided that $g$ is a bivariate polynomial, we investigate solutions of the delay differential equation by expanding them into Dirichlet series. Coefficients and arguments of these series are derived by means of a recurrence relation and their index set is isomorphic to a subset of planar graphs. Convergence results for these Dirichlet series rely heavily upon the derivation of generating functions of such graphs, counted with respect to certain recursively-defined functionals. We prove existence and convergence of Dirichlet series under different general conditions, thereby deducing much useful information about global behaviour of the solution.
\end{abstract}

\section{INTRODUCTION}

The conceptual point of departure for this paper is a proportional-delay variant of the familiar Riccati equation, namely

$$
y^{\prime}(t)=b y(t)+a y(q t)(1-y(q t)), \quad y(0)=y_{0},
$$

where $a, b, y_{0} \in \mathbb{C}$ and $q>0, q \neq 1$. Note that $q \in(0,1)$ yields a retarded equation, whereas $q>1$ results in an advanced equation. Our main interest is in the retarded case, but, as some of the results can be readily extended to advanced equations and others are valid only in the advanced case, we present them without restricting $q>0$.

Existence and uniqueness of the solution of (1.1) follows easily in the case $q \in(0,1)$ by replicating the proof of the familiar Picard-Lindelöf theorem. On the other hand, the existence of the solution in the advanced case is wide open and a matter of conjecture. It is known, however, that even in the linear case $y^{\prime}(t)=b y(t)+a y(q t)$ there might be an infinite number of solutions in the advanced case $q>1$ [5].

Received by the editors January 27, 1993.

1991 Mathematics Subject Classification. Primary 34K05; Secondary 34K20. 
Our interest lies in qualitative features of the solution of (1.1):

- Uniform boundedness: $\max _{t \geq 0}|y(t)|<\infty$;

- Asymptotic stability: $\lim _{t \rightarrow \infty} y(t)=0$;

- Periodicity: There exists $T>0$ such that $y(t+T)=y(t)$ for all $t \geq 0$;

- Almost periodicity: For every $\varepsilon>0$ there exists $T_{\varepsilon}, \liminf _{\varepsilon \rightarrow 0+} T_{\varepsilon}>0$, such that $\left|y\left(t+T_{\varepsilon}\right)-y(t)\right|<\varepsilon$ for all $t \geq 0$.

Frequently we are able to present our results in a considerably more general framework. Thus, in $\S 2$ we explore the existence of periodic solutions to the equation

$$
y^{\prime}(t)=f(y(t))+p(y(q t)), \quad y(0)=y_{0},
$$

where $f$ is an analytic function and $p$ is a polynomial. As long as $q<1$, existence and uniqueness of the solution can be again justified by extending the Picard-Lindelöf theorem. We prove in $\S 2$ that, unless $q$ is a rational number of the form $\frac{k}{l}$, where $l \in\{1,2, \ldots, \operatorname{deg} p\}$, the equation (1.2) has no periodic solutions. In the special case $(1.1)$ we have $p(x)=\operatorname{ax}(1-x)$, hence $q$ must be either an integer or half an integer. Moreover, if $f$ is, like in (1.1), a linear function then we prove that necessarily $k=1, l=\operatorname{deg} p$. Frequently we can prove this to be the best possible result. For example, letting $a, b \in \mathbb{R}$, $-\frac{1}{4}<\frac{b}{a}<\frac{1}{2}$, we prove that there exists a periodic solution to (1.1) when $q=\frac{1}{2}$.

A particularly useful means to analyse the behaviour of delay differential equations with the lag function $\theta(t)=q t$ is an expansion into Dirichlet series $[3,4]$. Section 3 is devoted to brief introduction into global analysis of Dirichlet series. This analysis is put into effect in $\S 4$, to explore solutions of

$$
y^{\prime}(t)=h(y(t), y(q t)), \quad y(0)=y_{0},
$$

where $h$ is a bivariate polynomial of total degree $m$ and, without loss of generality, $h(0,0)=0$. The Dirichlet expansion of (1.3) reads

$$
y(t)=\sum_{T \in \mathbb{T}} d_{T} e^{b \lambda_{T} t},
$$

where $b \in \mathbb{C} \backslash\{0\}$ and $\mathbb{T}$ is a countable set of indices. The coefficients $d_{T}$ and arguments $\lambda_{T}$ can be defined by nonlinear recursion. Needless to say, (1.4) is a formal expression, and our focus is on its convergence for $t \geq 0$. The main step in our analysis is in establishing that a very good choice of the index set $\mathbb{T}$ is a subset of connected planar trees. With this interpretation, the nonlinear recurrence is reduced to operation on graphs. Moreover, the question of convergence is subsumed in the problem of analyticity of generating functions, that count elements of $\mathbb{T}$ with certain features. In other words, we use graph theory to explicate the behaviour of a functional differential equation and algebraic combinatorics to analyse the aforementioned graphs...

The theory of $\S 4$ requires that the set $\left\{\lambda_{T}: T \in \mathbb{T} \backslash\left\{T_{\circ}\right\}\right\}$, where $T_{\circ}$ is the unique tree with one vertex, be bounded away from 1 . In $\S 5$ we explore a variety of different equations (1.3) in regard to that feature.

The theory of this paper can be extended to more elaborate equations and in $\S 6$ we focus on the rational equation

$$
y^{\prime}(t)=\frac{b y(t)}{1+a y(q t)}, \quad y(0)=y_{0}
$$

where $a, b, y_{0} \in \mathbb{C}$. 
The approach of Dirichlet series has many advantages-knowledge of a Dirichlet expansion is sufficient to explicate global behaviour of the solution, provide realistic bounds on its growth and even help in its numerical modelling -but also a critical shortcoming. To initialize the recursive procedure $\left\{d_{T}\right\}_{T \in \mathbb{T}_{m}}$, we need to assign a value to a single coefficient, $d_{T_{0}}$, say. This leads to an expansion (1.4) with the initial condition $y(0)=\sum_{T \in T_{m}} d_{T}$. In other words, the procedure produces a solution of (1.3) with some initial condition but the theory falls short of proving that the 'right' value of $d_{T}$ can be selected for every $y_{0}$ in a specific subset of the complex plane. The most we can claim is the existence of $y^{*}>0$ such that $\left|y_{0}\right|<y^{*}$ results in a convergent Dirichlet expansion. However, since $y^{*}$ is defined by restricting the magnitude of $d_{T_{\circ}}$, it is provided only in an implicit fashion.

This paper lies no claims to resolve completely the question of the global behaviour of even the simplest delay Riccati equation (1.1). For example, we can prove that the zero solution is stable, say, for specific regimes of $a, b \in \mathbb{C}$, but fall short of determining the basin of attraction with respect to $y_{0} \in \mathbb{C}$. Interestingly enough, numerical modelling by standard discretizations (in the retarded case $q \in(0,1))$ affirms that stability is lost when $\left|y_{0}\right|$ becomes too large. This implies that our analysis is on the right track and delivers the right kind of qualitative information, but much remains to be done to resolve the problem completely.

In line with $\S 6$, our theory can be extended to a wider range of similar problems. It requires a degree of care, since generality tends to interfere with convergence estimates. Nevertheless, it may lead to powerful new results and will undoubtedly be a subject for future research.

\section{Periodic SOlutions}

The theme of this section is the existence-or otherwise-of periodic solutions to the equation

$$
y^{\prime}(t)=f(y(t))+p(y(q t)), \quad y(0)=y_{0} \in \mathbb{C},
$$

where $f$ is a given analytic function, $p$ is an $m$ th degree complex polynomial and $q>0$. Let us assume that $(2.1)$ possesses a nonconstant periodic solution and that $T>0$ is the minimal period. Thus, $y(t+T) \equiv y(t), t \geq 0$, and this implies $y^{\prime}(t+T)-f(y(t+T)) \equiv y^{\prime}(t)-f(y(t))$. We now invoke (2.1) to argue that $p(y(q(t+T))) \equiv p(y(q t)), t \geq 0$. Changing the variable $q t \mapsto t$, we have

$$
p(y(t+q T)) \equiv p(y(t)), \quad t \geq 0 .
$$

Lemma 1. There exist positive integers $r \in\{1,2, \ldots, m\}$ and $w$ such that $q=\frac{w}{r}$.

Proof. Let $x_{k}(t):=y(t+k q T), k \in \mathbb{Z}^{+}$, and $p(z)=\sum_{l=0}^{m} p_{l} z^{l}, p_{m} \neq 0$. Our intermediate aim is to prove that there exists $s \in\{1,2, \ldots, m\}$ such that $x_{s} \equiv x_{0}$.

We rewrite $(2.2)$ as

$$
\sum_{l=0}^{m-1} p_{l+1}\left(x_{1}^{l+1}-x_{0}^{l+1}\right) \equiv 0 \text {. }
$$


Since $f$ is analytic, so is $y$ and we may deduce from (2.3) that either $x_{1} \equiv x_{0}$, $t \geq 0$, or

$$
\sum_{l=0}^{m-1} p_{l+1} \sum_{\substack{i_{0}+i_{1} \geq 0 \\ i_{0}+i_{1}=l}} x_{0}^{i_{0}} x_{1}^{i_{1}} \equiv 0
$$

We continue by induction. Thus, suppose that, for some $s \in\{1,2, \ldots, m-$ $1\}$, it is true that $x_{k} \not \equiv x_{0}, k=1,2, \ldots, s$, and that

$$
\sum_{l=0}^{m-s} p_{l+s} \sum_{\substack{i_{0}, i_{1}, \ldots, i_{s} \geq 0 \\ i_{0}+i_{1}+\cdots+i_{s}=l}} x_{0}^{i_{0}} x_{1}^{i_{1}} \cdots x_{s}^{i_{s}} \equiv 0 .
$$

Note that we have already seen that, for $s=1, x_{1} \not \equiv x_{0}$ implies (2.4).

Since $x_{k}(t)=y(t+k q T), k \in \mathbb{Z}^{+}$, we are allowed to step the indices of $x_{0}, \ldots, x_{s}$ up in (2.4) by one unit, hence

$$
\sum_{l=0}^{m-s} p_{l+s} \sum_{\substack{i_{0}, i_{1}, \ldots, i_{s}>0 \\ i_{0}+i_{1}+\cdots+i_{s}=l}} x_{1}^{i_{1}} \cdots x_{s}^{i_{s}} x_{s+1}^{i_{0}} \equiv 0 .
$$

Subtracting (2.4) from the latter identity yields

$$
\sum_{l=0}^{m-s-1} p_{l+s+1} \sum_{\substack{i_{0}, i_{1}, \ldots, i_{s} \geq 0 \\ i_{0}+i_{1}+\cdots+i_{s}=l}}\left(x_{s+1}^{i_{0}+1}-x_{0}^{i_{0}+1}\right) x_{1}^{i_{1}} \cdots x_{s}^{i_{s}} \equiv 0 .
$$

Clearly, $x_{s+1} \equiv x_{0}$ is a solution of (2.5). Suppose, however, that $x_{s+1} \not \equiv x_{0}$. Since $y$ is analytic, so is $x_{k}, k \in \mathbb{Z}^{+}$. Therefore,

$$
\sum_{l=0}^{m-s-1} p_{l+s+1} \sum_{\substack{i_{0}, i_{1}, \ldots, i_{s} \geq 0 \\ i_{0}+i_{1}+\cdots,+i_{s}=l}} \frac{x_{s+1}^{i_{0}+1}(t)-x_{0}^{i_{0}+1}(t)}{x_{s+1}(t)-x_{0}(t)} x_{1}^{i_{1}}(t) \cdots x_{s}^{i_{s}}(t)=0
$$

holds for $t \in \mathscr{T} \subseteq[0, \infty)$, where $\mathscr{T}$ is a continuum. Thus,

$$
\sum_{l=0}^{m-s-1} p_{l+s+1} \sum_{\substack{i_{0}, i_{1}, \ldots, i_{s}>0 \\ i_{0}+i_{1}+\cdots,+i_{s}=l}} \frac{x_{s+1}^{i_{0}+1}-x_{0}^{i_{0}+1}}{x_{s+1}-x_{0}} x_{1}^{i_{1}} \cdots x_{s}^{i_{s}} \equiv 0 .
$$

Trivial manipulation affirms that this is identical to (2.4) with $s$ replaced by $s+1$. This advances the induction one step.

The identity (2.4) cannot be true for $s=m$, since $p_{m} \neq 0$ and $y \not \equiv 0$. Thus, we deduce that $x_{r} \equiv x_{0}$ for some $r \in\{1,2, \ldots, m\}$. In other words, there exists $1 \leq r \leq m$ such that

$$
y(t+r q T)=y(t), \quad t \geq 0,
$$

and $y$ is periodic of both period $T$ and period $r q T$. However, it is trivial to verify that if a function $y$ is periodic of distinct periods $T_{1}$ and $T_{2}$, say, then it possesses period $\left|T_{2}-T_{1}\right|$. Consequently, and unless $r q$ is an integer, $y$ is periodic of period $(q r-[q r]) T \in(0, T)$, and this contradicts our assumption that $T$ is the minimal period. Hence, $q$ is a rational number of the form stipulated in the statement of the lemma. 
Lemma 2. Let $w$ and $r$ be relatively prime. In the special case $f(z)=f_{0}+f_{1} z$, i.e.

$$
y^{\prime}(t)=f_{0}+f_{1} y(t)+p(y(q t)), \quad y(0)=y_{0},
$$

necessarily $w=1$ in Lemma 1 . In other words, periodicity implies that $q=\frac{1}{r}$ for some $r \in\{1,2, \ldots, m\}$.

Proof. We assume again that $T>0$ is the minimal period. Since $y$ is analytic, so is its derivative. Hence the Fourier expansions of $y$ and of $y^{\prime}$ are both pointwise convergent [6] and

$$
y(t)=\sum_{n \in \mathbb{Z}} d_{n} \exp \frac{2 \pi i n t}{T}, \quad t \geq 0 .
$$

Substitution into (2.1) yields

$$
\begin{aligned}
\psi(t) & :=y^{\prime}(t)-f(y(t))=\sum_{n \in \mathbb{Z}} \psi_{n} \exp \frac{2 \pi i n t}{T} \\
& =-f_{0}+\sum_{n \in \mathbb{Z}}\left(\frac{2 \pi i n t}{T}-f_{1}\right) d_{n} \exp \frac{2 \pi i n t}{T} \\
& =p_{0}+\sum_{l=1}^{p} p_{l} \sum_{k_{1}, k_{2}, \ldots, k_{l} \in \mathbb{Z}} d_{k_{1}} d_{k_{2}} \cdots d_{k_{l}} \exp \frac{2 \pi i\left(k_{1}+\cdots+k_{l}\right) w t}{r T} .
\end{aligned}
$$

Comparing coefficients in (2.7), we deduce that for every $n \in \mathbb{Z}$ either $\psi_{n}=0$ or there exist $l \in\{1,2, \ldots, m\}$ and $k_{1}, k_{2}, \ldots, k_{l} \in \mathbb{Z}$ such that

$$
r n=w \sum_{j=1}^{l} k_{j} .
$$

$w$ and $r$ being relatively prime, the latter implies that $w$ divides $n$. Hence

$$
\psi(t)=\sum_{n \in \mathbb{Z}} \psi_{n w} \exp \frac{2 \pi i n w t}{T}
$$

and it is periodic of period $\frac{T}{w}$. Our contention is that so is $y$. Because of (2.7), it is clear that, unless $n$ is divisible by $w$,

$$
\left(\frac{2 \pi i n}{T}-f_{1}\right) d_{n}=0
$$

Thus, for this range of $n$, necessarily $d_{n}=0$, except that there might exist a unique $\tilde{n}$, say, such that $f_{1}=2 \pi i \tilde{n} / T$. We deduce that

$$
y(t)=\sum_{n \in \mathbb{Z}} d_{w n} \exp \frac{2 \pi i n w t}{T}+d_{\tilde{n}} \exp \frac{2 \pi i \tilde{n} t}{T} .
$$

Thus,

$$
y\left(t+\frac{T}{w}\right)-y(t)=\beta \exp \frac{2 \pi i \tilde{n} t}{T},
$$

where

$$
\beta:=\exp \frac{2 \pi i \tilde{n}}{w T}-1 .
$$


Iterating (2.8) we obtain

$$
y(t+T)=y(t)+w \beta \exp \frac{2 \pi i \tilde{n} t}{T} .
$$

Since $T$ is a period, it follows that $\beta=0$. Consequently, $y$ is also periodic of period $\frac{T}{w}$. Unless $w=1$, this contradicts the assumption that $T>0$ is the minimal period.

Lemmas 1 and 2 can be combined to spell out the main result of this section.

Theorem 3. The equation

$$
y^{\prime}(t)=f(y(t))+p(y(q t)), \quad y(0)=y_{0} \in \mathbb{C},
$$

where $q>0, f$ is an analytic function and $p$ is an mth degree polynomial, possesses a periodic nonconstant solution only if $q$ is a rational number of the form $q=\frac{w}{r}$, where $r \in\{1,2, \ldots, m\}$. Moreover, if $f$ is a linear function then $w=1$.

The result of Theorem 3 is frequently the best possible. Thus, suppose that $p$ is quadratic in (2.6). In that case (and excluding the no-delay case $q=1$ ) Lemma 2 leaves out just one choice of $q$, namely $q=\frac{1}{2}$. The equation reads

$$
y^{\prime}(t)=f_{0}+f_{1} y(t)+p_{1} y\left(\frac{t}{2}\right)+p_{2} y^{2}\left(\frac{t}{2}\right), \quad p_{2} \neq 0 .
$$

Letting $z(t):=y(t)+\frac{1}{2}\left(1+p_{1}\right), t \geq 0$, we obtain

$$
z^{\prime}(t)=\alpha_{0}+\alpha_{1} z(t)+\beta z\left(\frac{t}{2}\right)\left(1-z\left(\frac{t}{2}\right)\right),
$$

where

$$
\alpha_{0}=f_{0}-\frac{1}{2}\left(1+p_{1}\right)\left(f_{1}+p_{1}\right)+\frac{1}{4}\left(1+p_{1}\right)^{2} p_{2}, \quad \alpha_{1}=f_{1}, \quad \beta=-p_{2} .
$$

We seek a solution of the form

$$
z(t)=\frac{1}{2}+a \cos \lambda t+b \sin \lambda t .
$$

Substitution in (2.9) and comparison of coefficients results, after some manipulation, in

$$
\begin{gathered}
\alpha_{0}+\frac{1}{2} \alpha_{1}+\frac{1}{4} \beta=\frac{1}{2} \beta\left(a^{2}+b^{2}\right), \\
b \lambda=\alpha_{1} a-\frac{1}{2} \beta\left(a^{2}-b^{2}\right), \quad-a \lambda=\alpha_{1} b-\beta a b .
\end{gathered}
$$

It is straightforward to verify that the solution is

$$
\lambda=\frac{1}{2} \beta b, \quad a=2-\frac{\alpha_{1}}{\beta}, \quad b= \pm\left\{2 \frac{\alpha_{0}}{\beta}+\left(1+4 \frac{\alpha_{1}}{\beta}\right)\left(\frac{1}{2}-\frac{\alpha_{1}}{\beta}\right)\right\}^{1 / 2} .
$$

Needless to say, we require for periodicity a real value of $\lambda$, and this is the case if and only if

$$
2 \frac{\alpha_{0}}{\beta}+\left(1+4 \frac{\alpha_{1}}{\beta}\right)\left(\frac{1}{2}-\frac{\alpha_{1}}{\beta}\right)>0
$$

In the special case $\alpha_{0}=0$, periodic solution of the stipulated form exists for

$$
-\frac{1}{4}<\frac{\alpha_{1}}{\beta}<\frac{1}{2}
$$


For example,

$$
\begin{aligned}
& z^{\prime}(t)=\frac{1}{4} z(t)+z\left(\frac{t}{2}\right)\left(1-z\left(\frac{t}{2}\right)\right) \Rightarrow z(t)=\frac{1}{2}+\frac{1}{2} \cos \frac{\sqrt{2} t}{4}+\frac{\sqrt{2}}{2} \sin \frac{\sqrt{2} t}{4} \\
& z^{\prime}(t)=-\frac{1}{8} z(t)+z\left(\frac{t}{2}\right)\left(1-z\left(\frac{t}{2}\right)\right) \Rightarrow z(t)=\frac{1}{2}-\frac{1}{4} \cos \frac{\sqrt{5} t}{8}+\frac{\sqrt{5}}{4} \sin \frac{\sqrt{5} t}{8} .
\end{aligned}
$$

Of course, we make no claim that these are the only periodic solutions of (2.9), that (2.10) represents the broadest possible range of parameters giving rise to periodic $z$ or that the only initial value that is consistent with periodicity is $z(0)=\frac{1}{2}+2 \alpha_{1} / \beta$. Numerical results, however, support the conjecture that all these statements might be true.

Similar construction can be sometimes extended to higher degree equations. For example, long and tedious calculation verifies that the equation

$$
y^{\prime}(t)=p_{0}+p_{1} y\left(\frac{t}{4}\right)+p_{2} y^{2}\left(\frac{t}{4}\right)+p_{3} y^{3}\left(\frac{t}{4}\right)+p_{4} y^{4}\left(\frac{t}{4}\right), \quad p_{4} \neq 0,
$$

where

$$
p_{0}=\frac{1}{8} \frac{p_{2}^{2}}{p_{4}}-\frac{1}{32} \frac{p_{2} p_{3}^{2}}{p_{4}^{2}}-\frac{1}{512} \frac{p_{3}^{4}}{p_{4}^{3}}, \quad p_{1}=\frac{1}{2} \frac{p_{2} p_{3}}{p_{4}}-\frac{1}{8} \frac{p_{3}^{3}}{p_{4}^{2}},
$$

and provided that $3 p_{3}^{2}>8 p_{2} p_{4}$, possesses the periodic solution $y(t)=a+$ $b \sin \lambda t$ with

$$
a=-\frac{p_{3}}{4 p_{4}}, \quad b= \pm \frac{1}{p_{4}}\left\{\frac{3}{8} p_{3}^{2}-p_{2} p_{4}\right\}^{1 / 2}, \quad \lambda= \pm \frac{1}{8 p_{4}^{2}}\left\{\frac{3}{8} p_{3}^{2}-p_{2} p_{4}\right\}^{3 / 2} .
$$

Computer experiments indicate that a periodic solution of (2.9), when it exists, is of a different nature to the familiar periodic solutions of ordinary differential equations. Firstly, it is unstable in the initial value and any $z(0)=z_{0} \neq$ $\frac{1}{2}+2 \alpha_{1} / \beta, \alpha_{0}+\alpha_{1} z_{0}+\beta z_{0}\left(1-z_{0}\right) \neq 0$, appears to lead to an oscillatory solution with asymptotically growing amplitude, which cannot be uniformly bounded. Secondly, it is typical of periodic solutions of ordinary differential equations, parametrized by their parameters, to occur at a Hopf bifurcation, when the behaviour changes from an unstable to stable oscillation (or, in a phase space, from an outward-oriented spiral to an inward-oriented spiral). As indicated by numerical modelling, this is not the case for (2.9), when the parameter $\alpha_{1}$, say, is varied within a regime consistent with (2.10). If this is done for fixed $z(0)$ then periodicity occurs at a 'transition' from an unstable spiral to another unstable spiral. On the other hand, for every such $\alpha_{1}$ there exists, by our analysis, an initial value that gives rise to periodicity-in this strict sense, periodic behaviour is robust.

Periodicity and tendence to a limit (the latter being the main theme in the sequel) are not the only phenomena that can be encountered in the context of delay equations. It is known [4] that the solution of the linear equation

$$
y^{\prime}(t)=i a y(t)+b y(q t), \quad y(0) \neq 0,
$$

where $a \in \mathbb{R} \backslash\{0\}, b \in \mathbb{C},|b|<|a|$ and $q \in(0,1)$, is an almost periodic function [6]. Another phenomenon occurs when the equation (2.9) is solved at the left end of the range (2.10), namely when $\alpha_{1}=-\frac{1}{4} \beta$. Computer experimentation indicates that, at least for $\alpha_{0}=0, \beta>0$ and $z(0) \in\left(0, \frac{3}{4}\right)$, the solution exhibits chaotic character. Figure 1 displays the solution of

$$
z^{\prime}(t)=-\frac{1}{4} z(t)+z\left(\frac{t}{2}\right)\left(1-z\left(\frac{t}{2}\right)\right), \quad z(0)=\frac{1}{2} .
$$




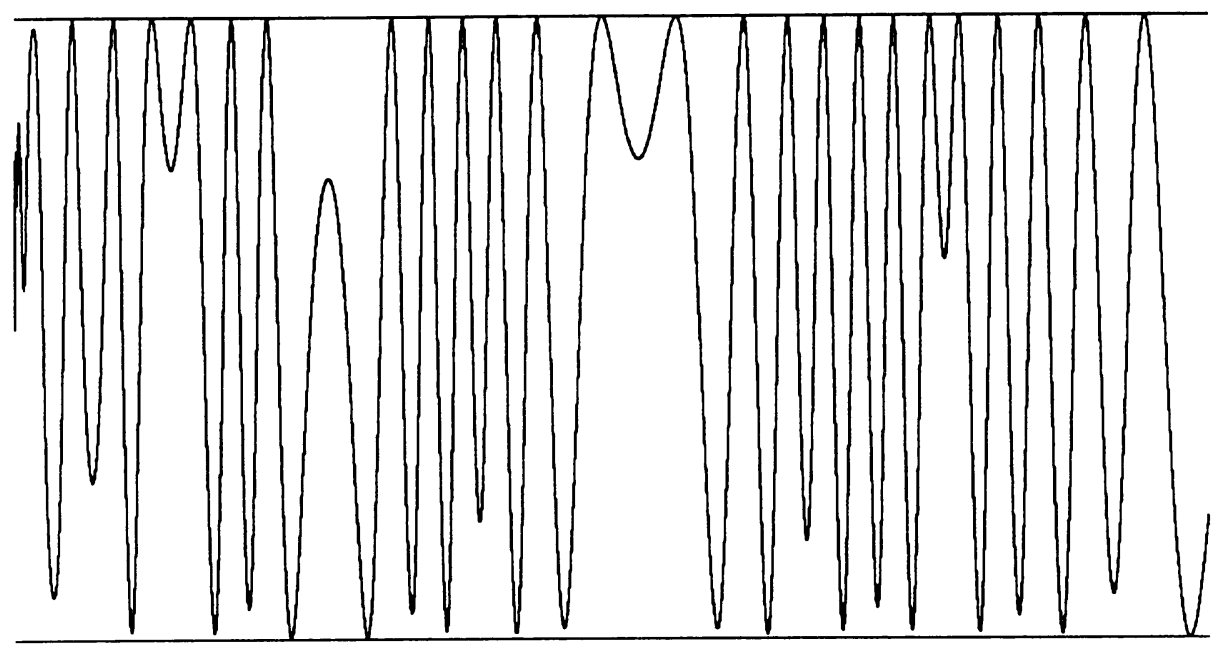

FIGURE 1. The solution of $z^{\prime}(t)=-\frac{1}{4} z(t)+z(t / 2)$ $\cdot(1-z(t / 2)), z(0)=\frac{1}{2}$, for $0<t<10^{5}$. Horizontal lines denote $z \equiv 0$ and $z \equiv 1$

The exact nature of the solution of (2.11) —and of solutions of similar equations -is at present a subject of ongoing research.

\section{DiRICHLET EXPANSIONS}

A Dirichlet expansion is (in general, countably infinite) linear combination of exponentials [3]. Let

$$
f(t):=\sum_{T \in \mathbb{T}} d_{T} e^{b \lambda_{T} t}, \quad t \geq 0,
$$

where $b \in \mathbb{C} \backslash\{0\}, \lambda_{T}>0$ and $d_{T} \in \mathbb{C}$ for all $T$ in a countable set $\mathbb{T} .{ }^{1}$ We assume that

$$
d^{*}:=\sum_{T \in \mathbb{T}}\left|d_{T}\right|<\infty .
$$

The purpose of the present section is to provide a brief review of asymptotic features of Dirichlet series that are of interest in the remainder of this paper. We do not dwell on the more usual applications of Dirichlet series, e.g. in number theory [3].

Let $\lambda^{*}:=\liminf _{T \in \mathbb{T}} \lambda_{T} \geq 0$. It follows at once from (3.1) and (3.2) that

$$
|f(t)| \leq d^{*} e^{\operatorname{Re} b \lambda^{*} t}, \quad t \geq 0 .
$$

We deduce that $(3.1)$ is well defined and bounded for all $t \geq 0$. Moreover, $\lambda^{*}>0$ and $\operatorname{Re} b<0$ imply that $\lim _{t \rightarrow \infty} f(t)=0$ and $f$ is asymptotically stable. However, $\lambda^{*}>0$ is not necessary for asymptotic stability. The following result generalizes a method of proof implicit in [3] to cater for the case $\lambda^{*}=0$.

${ }^{1}$ Of course, we may assume without loss of generality that the index set $\mathbb{T}$ coincides with $\mathbb{Z}^{+}$. This, however, is unhelpful to the analysis in the sequel. We demonstrate in $\S \S 3$ and 4 that a more 'exotic' form of $\mathbb{T}$ is advantageous, since it is more in tune with the recursive technique of implicitly defining the arguments $\lambda_{T}, T \in \mathbb{T}$. 
Proposition 4. Let $\operatorname{Re} b<0$. Then $\lim _{t \rightarrow \infty} f(t)=0$ and $f$ is asymptotically stable.

Proof. We wish to show that for every small $\varepsilon>0$ there exists $t_{\varepsilon}>0$ such that

$$
|f(t)|<\varepsilon, \quad t>t_{\varepsilon}
$$

Since the sequence $\left\{d_{T}\right\}_{t \in \mathbb{T}}$ is $l_{1}$, there exists a partition of $\mathbb{T}=\mathbb{T}_{1} \cup \mathbb{T}_{2}$ such that $\mathbb{T}_{1} \cap \mathbb{T}_{2}=\varnothing, \mathbb{T}_{2}$ is finite and

$$
\sum_{T \in \mathbb{T}_{2}}\left|d_{T}\right|>d^{*}-\frac{\varepsilon}{2}
$$

The latter inequality, $\lambda^{*} \geq 0$ and $\operatorname{Re} b<0$ imply that

$$
\left|\sum_{T \in \mathbb{T}_{1}} d_{T} e^{b \lambda_{T} t}\right| \leq \sum_{T \in \mathbb{T}_{1}}\left|d_{T}\right|<\frac{\varepsilon}{2}, \quad t \geq 0
$$

Since $\mathbb{T}_{2}$ is finite, necessarily $\lambda^{(\varepsilon)}:=\min _{T \in \mathbb{T}_{2}} \lambda_{T}>0$. Consequently,

$$
\left|\sum_{T \in \mathbb{T}_{2}} d_{T} e^{b \lambda_{T} t}\right| \leq \sum_{T \in \mathbb{T}_{2}}\left|d_{T}\right| e^{\operatorname{Re} b \lambda^{(e)} t} \leq d^{*} e^{\operatorname{Re} b \lambda^{(e)} t}, \quad t \geq 0 .
$$

Thus, choosing

$$
t_{\varepsilon}:=\frac{\log \varepsilon /\left(2 d^{*}\right)}{\lambda^{(\varepsilon)} \operatorname{Re} b},
$$

(3.5) and (3.6) imply the inequality (3.4). Hence asymptotic stability.

The behaviour of Dirichlet series is known also on their stability boundary.

Proposition 5. If $\operatorname{Re} b=0$ then $y$ is almost periodic for $t \geq 0$.

Proof. According to a theorem of $\mathrm{H}$. Bohr, almost periodic functions are $L_{\infty}[0, \infty)$ limits of trigonometric polynomials [6]. In other words, $y$ is almost periodic if and only if there exist functions $\left\{y_{n}\right\}_{n=1}^{\infty}$ such that

$$
y^{[n]}(t)=\sum_{T \in \mathbb{T}_{n}} d_{T}^{[n]} e^{b \lambda_{T}^{[n]} t}, \quad n \in \mathbb{Z}^{+},
$$

where $\mathbb{T}_{n}$ is of finite cardinality, and $\lim _{n \rightarrow \infty} y^{[n]}(t)=y(t)$ uniformly for all $t \geq 0$.

Since $\sum_{T}\left|d_{T}\right|=d^{*}<\infty$, we can choose finite sets $\left\{\mathbb{T}_{n}\right\}_{n=1}^{\infty}$ such that

$$
\sum_{T \in \mathbb{T}_{n}}\left|d_{T}\right|>d^{*}-\frac{1}{n}, \quad n=1,2, \ldots
$$

We let

$$
y^{[n]}(t):=\sum_{T \in \mathbb{T}^{n]}} d_{T} e^{b \lambda_{T} t}, \quad n=1,2, \ldots
$$

Let $\mathbb{S}^{[n]}:=\mathbb{T} \backslash \mathbb{T}^{[n]}$. Then $\operatorname{Re} b=0$ implies that

$$
\left|y(t)-y_{n}(t)\right| \leq \sum_{T \in \mathbf{S}[n]}\left|d_{T}\right|<\frac{1}{n}
$$


uniformly for $t \geq 0$. This implies that $y$ is almost periodic.

It has been demonstrated in [4] that the linear vector equation

$$
\mathbf{y}^{\prime}(t)=A \mathbf{y}(t)+B \mathbf{y}(q t), \quad \mathbf{y}(0)=\mathbf{y}_{0},
$$

possesses an almost periodic Dirichlet series, provided that $A$ is invertible, $\max \operatorname{Re} \sigma(A)=0, \rho\left(A^{-1} B\right)<1$, and $\mathbf{y}_{0}$ is not orthogonal to the space spanned by the eigenvectors of pure imaginary eigenvalues of $A$. Much depends on whether $q \in(0,1)$ is rational, since in the latter case there exist rotational almost symmetries in the 'phase space' $(\operatorname{Re} y, \operatorname{Im} y)$. Many geometric features of such solutions are reported in [4], others are subject of an ongoing research.

4. General equations $y^{\prime}(t)=h(y(t), y(q t)), h$ a POlynOMial

It is easy to formulate necessary conditions for asymptotic stability for delay differential equations, by following techniques intimately related to the standard variational equation approach. Thus, consider the equation

$$
y^{\prime}(t)=h(y(t), y(q t)), \quad y(0)=y_{0} \in \mathbb{C} .
$$

As long as $q \in(0,1)$ and $h$ is locally Lipschitz in each of its variables, existence and uniqueness of the solution can be deduced by standard methods. Moreover, the set of all fixed points of (4.1) coincides with $\mathscr{H}:=\{\hat{y} \in \mathbb{C}: h(\hat{y}, \hat{y})=0\}$. Suppose that $h$ is smoothly differentiable in both variables and that $\hat{y} \in \mathscr{H}$ is attractive. In other words, there exists $\delta>0$ such that $\left|y_{0}-\hat{y}\right|<\delta$ implies that $\lim _{t \rightarrow \infty} y(t)=\hat{y}$. Let $\varepsilon(t):=y(t)-\hat{y}$ and suppose that $t$ is sufficiently large, so that $|\varepsilon(t)|<\eta$ for all $t \geq q t_{0}$. Substitution into (4.1) yields

$$
\varepsilon^{\prime}(t)=h_{1}(\hat{y}, \hat{y}) \varepsilon(t)+h_{2}(\hat{y}, \hat{y}) \varepsilon(q t)+\mathscr{O}\left(\eta^{2}\right), \quad t \geq t_{0} .
$$

Here $h_{1}$ and $h_{2}$ are the derivatives of $h$ with respect to the first and the second variable, respectively.

Truncation of the $\mathscr{O}\left(\eta^{2}\right)$ term in (4.2) results in the pantograph equation

$$
v^{\prime}(t)=b v(t)+a v(q t),
$$

where $b=h_{1}(\hat{y}, \hat{y})$ and $a=h_{2}(\hat{y}, \hat{y})$. Asymptotic behaviour of $(4.3)$ has been determined in [4]:

1. If $\operatorname{Re} b<0$ and $|a|<|b|$ then $\lim _{t \rightarrow \infty} v(t)=0$;

2. If either $\operatorname{Re} b>0$ or $|a|>|b|$ then, as long as $v(0) \neq 0, \lim \sup _{t \rightarrow \infty}|v(t)|$ $=\infty$

3. If $\operatorname{Re} b=0$ and $|a| \leq|b|$ then $v$ is almost periodic;

4. If $\operatorname{Re} b<0$ and $|a|=|b|$ then, under a logarithmic change of variables, $v$ tends to an almost periodic curve. (The latter case has not been derived in [4]. It will feature in a future paper.)

Hence, if $\operatorname{Re} h_{1}(\hat{y}, \hat{y})>0$ or $\left|h_{2}(\hat{y}, \hat{y})\right|>\left|h_{1}(\hat{y}, \hat{y})\right|$ then it is impossible for $\varepsilon$ to tend to zero in (4.2).

Theorem 6. A necessary condition for $\hat{y} \in \mathscr{H}$ being an asymptotically stable fixed point of (4.1) is that $\operatorname{Re} h_{1}(\hat{y}, \hat{y}) \leq 0$ and $\left|h_{2}(\hat{y}, \hat{y})\right| \leq\left|h_{1}(\hat{y}, \hat{y})\right|$.

It is important to emphasize that there is nothing in our analysis to support the view that the condition of Theorem 6 is sufficient for stability. The remainder of this paper is devoted in the main to the exploration of sufficient conditions for asymptotic stability. 
We next assume that $h$ is a bivariate polynomial of total degree $m$,

$$
h(u, v)=\sum_{k=0}^{m} \sum_{l=0}^{k} a_{k, l} u^{l} v^{k-l} .
$$

In the spirit of our discussion so far, we stipulate that $h(0,0)=0$ and that the fixed point of interest lies at the origin, otherwise we subject $y$ to a linear transformation. Finally, we require $b:=a_{1,1} \neq 0$. In other words, the focus of our attention is on the equation

$$
y^{\prime}(t)=b y(t)+a_{1,0} y(q t)+\sum_{k=2}^{m} \sum_{l=0}^{k} a_{k, l} y^{l}(t) y^{k-l}(q t), \quad y(0)=y_{0}, b \neq 0
$$

Subsitution of the Dirichlet solution (3.1), that is $y(t)=\sum_{T \in \mathbb{T}} d_{T} e^{b \lambda_{T} t}$, into (4.4) yields

$$
\begin{aligned}
& b \sum_{T \in \mathbb{T}} d_{T}\left(\lambda_{T}-1\right) e^{b \lambda_{T} t}=a_{1,0} \sum_{T \in \mathbb{T}} d_{T} e^{b q \lambda_{T} t} \\
& \quad+\sum_{k=2}^{m} \sum_{l=0}^{k} a_{k, l} \sum_{T_{1}, \ldots, T_{k} \in \mathbb{T}} d_{T_{1}} d_{T_{2}} \cdots d_{T_{k}} \exp \left\{b\left(\sum_{i=1}^{l} \lambda_{T_{i}}+q \sum_{i=l+1}^{k} \lambda_{T_{i}}\right) t\right\} .
\end{aligned}
$$

As usual, an empty sum is assumed to be naught.

Let $\Lambda:=\left\{\lambda_{T}: T \in \mathbb{T}\right\}$. We need to choose $\Lambda$ so that each nonvanishing exponential on the right of (4.5) can be matched with such an exponential on the left. This gives rise to the following composition rules, which provide a recursive definition of $\Lambda$.

1. $1 \in \Lambda$;

2. If $k \in\{1,2, \ldots, m\}, l \in\{0,1, \ldots, k\},(k, l) \neq(1,1), a_{k, l} \neq 0$ and $\lambda_{T_{1}}, \lambda_{T_{2}}, \ldots, \lambda_{T_{k}} \in \Lambda$, then

$$
\sum_{i=1}^{l} \lambda_{T_{i}}+q \sum_{i=l+1}^{k} \lambda_{T_{i}} \in \Lambda
$$

Proposition 7. Each $\lambda \in \Lambda$ is a polynomial in $q$ with integer nonnegative coefficients.

Proof. Follows from the composition rules by straightforward induction.

It is highly useful to identify members of the index set $\mathbb{T}$ with multiply-rooted planar trees. We remind the reader that a tree is a connected graph $G=(V, E)$ where each two distinct vertices $v_{1}, v_{2} \in V$ are joined by precisely one path of edges from $E$ and it is planar if distinct trees with the same topology (e.g. mirror images) are counted separately $[1,2]$. For the purpose of this paper we define a multiply-rooted tree as the ordered pair $\langle G, R\rangle$, where the multiroot $R \subseteq V$ has the property that each vertex $v \in V \backslash R$ is linked to exactly one element of $R$ by exactly one path, that does not pass through any other vertex from $R .^{2}$ We call such a path the principal path of $v$ and extend this definition to $R$ by stipulating that the principal path of $v \in R$ is the zero-length path

${ }^{2}$ It is easy to prove that the restriction of $G$ to $R$ is itself a connected tree, consisting of a single path. This plays no role in our analysis and is left to the reader. 
consisting of $v$ itself. Principal paths define partial ordering on $\langle G, R\rangle$ (a trivial extension of a monotone ordering of a rooted tree).

The order of a graph is the number of its vertices. We denote the order of a multiply-rooted planar tree $T$ by ord $T$ and note that there exists a unique such tree of order $1, T_{\circ}$, say.

We say that $v \in V$ is a top vertex if it does not lie on any principal path of a vertex from $V \backslash\{v\}$. In other words, a top vertex has no children in the partial ordering of $\langle G, R\rangle$. Moreover, len $v$ denotes the length of the principal path of a top vertex $v \in V$.

Let $\mathrm{W}$ be the set of all multiply-rooted planar trees. We define two operations on elements of $\mathbb{W}$ :

Addition. Let $T_{i}=\left\langle G_{i}, R_{i}\right\rangle \in \mathbb{W}, G_{i}=\left(V_{i}, R_{i}\right), i=1,2, \ldots, s, s \geq 2$. We stipulate that $V_{i} \cap V_{j}=\varnothing$ for $i \neq j$. Then

$$
T=T_{1}-T_{2}-\cdots-T_{s}=\langle G, R\rangle, \quad G=(V, E),
$$

is defined as follows: $G=\bigcup_{i=1}^{s} G_{i}, R=\bigcup_{i=1}^{s} R_{i}$, whereas $E=\bigcup_{i=1}^{s} E_{i} \cup E_{*}$ and the set $E_{*}$ is made out of $s-1$ edges linking the rightmost element of $R_{i}$ to the leftmost element of $R_{i+1}, i=1,2, \ldots, s$. (Note that 'leftmost' and 'rightmost' are well defined for planar trees.) For example,

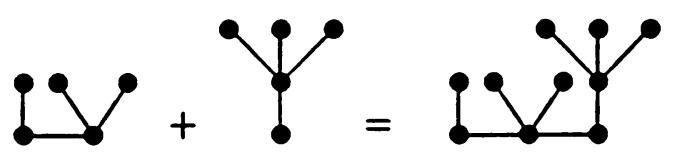

We observe that addition in $\mathbb{W}$ is not commutative and that $\operatorname{ord} T=\operatorname{ord} T_{1}+$ $\cdots+\operatorname{ord} T_{s}$.

Rooting. Given $T^{\prime} \in \mathbb{W}, T^{\prime}=\left\langle G^{\prime}, R^{\prime}\right\rangle, G^{\prime}=\left(V^{\prime}, E^{\prime}\right)$, we define

$$
T=\oint^{T^{\prime}}=\langle G, R\rangle, \quad G=(V, E)
$$

by letting $V=V^{\prime} \cup v$, say, where $v \notin V^{\prime}, R=\{v\}$ and $E=E_{\alpha} \cup E_{\beta}$, where $E_{\alpha} \subseteq E^{\prime}$ is made up by removing any edges that are wholly in $R^{\prime}$, whereas $E_{\beta} \subseteq\left(V^{\prime}, v\right)$ contains edges linking each element of $R^{\prime}$ with $v$. For example,
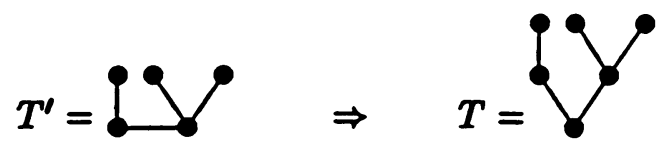

We note that ord $T=$ ord $T^{\prime}+1$.

It is trivial to verify that $\mathrm{W}$ is closed under addition and rooting.

We map $\mathrm{W}$ into the set of polynomials in $q$ with nonnegative integer coefficients by letting

$$
\lambda_{T}:=\sum_{v \text { is a top vertex of } T} q^{\operatorname{len} v} .
$$


Note that the map $T \mapsto \lambda_{T}$ is an injection: in general, many members of $\mathbb{W}$ are mapped to the same polynomial. We will return to this point in the sequel.

The composition rules may now be reformulated in tree terminology. Thus, $\mathbb{T}$ is constructed out of elements from $\mathbb{W}$ such that

1. $T_{\circ} \in \mathbb{T}$;

2. If $k \in\{1,2, \ldots, m\}, l \in\{0,1, \ldots, k\},(k, l) \neq(1,1), a_{k, l} \neq 0$ and $T_{1}, T_{2}, \ldots, T_{k} \in \mathbb{T}$ then

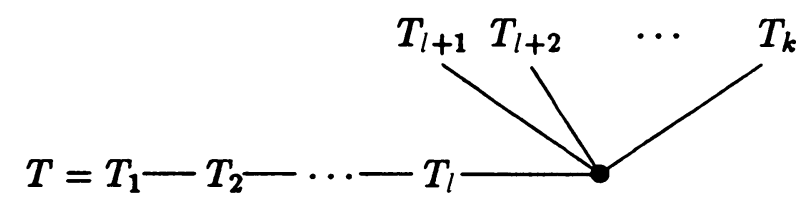

It follows at once from (4.7) that

$$
\lambda_{T}=\sum_{i=1}^{l} \lambda_{T_{i}}+q \sum_{i=l+1}^{k} \lambda_{T_{i}} .
$$

Hence, by (4.6), this construction corresponds to the second composition rule. We stress three important points. Firstly, not every element of $\mathbb{W}$ is bound to appear in $\mathbb{T}$. Secondly, many $\lambda_{T}$ 's (even a countable infinity) may be identical, although possibly with different coefficients $d_{T}$. Indeed, the same element of $\mathbb{W}$ might well feature several times in $\mathbb{T}$. Thirdly, the exact contents of $\mathbb{T}$ depend on $m$ and on the set

$$
\left\{(k, l): a_{k, l} \neq 0, k=1,2, \ldots, m, l=0,1, \ldots, k,(k, l) \neq(1,1)\right\} .
$$

These points, however cause no difficulty.

Let $\omega_{k, l}:=a_{k, l} / b, k=1,2, \ldots, m, l=0,1, \ldots, k,(k, l) \neq(1,1)$.

Proposition 8. There exist a function $\alpha: \mathbb{T} \rightarrow \mathbb{R}$, dependent just on $q$, and functions

$$
\beta, \gamma_{i, j}: \mathbb{T} \rightarrow \mathbb{Z}^{+}, \quad i=1,2, \ldots, m, j=0,1, \ldots, i,(i, j) \neq(1,1),
$$

independent of $q$ and the coefficients $a_{i, j}$, such that

$$
d_{T}=\frac{1}{\alpha(T)} d_{T_{\circ}}^{\beta(T)} \prod_{i=1}^{m} \prod_{\substack{j=0 \\(i, j) \neq(1,1)}}^{i} \omega_{i, j}^{\gamma_{i, j}(T)}, \quad T \in \mathbb{T} .
$$

Moreover, $\beta(T)$ is the number of top vertices of $T \in \mathbb{T}$.

Proof. Substitution of (4.10) into (4.5) affirms that the proposition is true, as well as providing explicit recurrence relations for the evaluation of the underlying functions. Again, we follow the composition rules.

1. $\alpha\left(T_{\circ}\right)=\beta\left(T_{\circ}\right)=1, \gamma_{k, l}\left(T_{\circ}\right)=0$ by definition.

2. Suppose that $T \in \mathbb{T}$, ord $T \geq 2$, is constructed from $T_{1}, T_{2}, \ldots, T_{k} \in \mathbb{T}$ as in (4.8). Then it follows from (4.5) that

$$
\left(\lambda_{T}-1\right) d_{T}=\omega_{k, l} \prod_{s=1}^{k} d_{T_{s}}
$$


and substituting (4.10) yields

$$
\begin{aligned}
\frac{1}{\alpha(T)} d_{T_{\circ}}^{\beta(T)} \prod_{i=1}^{m} \prod_{\substack{j=0 \\
(i, j) \neq(1,1)}}^{i} \omega_{i, j}^{\gamma_{i, j}(T)} & \frac{\omega_{k, l}}{\prod_{s=1}^{k} \alpha\left(T_{s}\right)} d_{T_{\circ}}^{\sum_{s=1}^{k} \beta\left(T_{s}\right)} \prod_{i=1}^{m} \prod_{\substack{j=0 \\
(i, j) \neq(1,1)}}^{i} \omega_{i, j}^{\sum_{s=1}^{k} \gamma_{i, j}\left(T_{s}\right)}
\end{aligned}
$$

Therefore

$$
\alpha(T)=\left(\lambda_{T}-1\right) \prod_{s=1}^{k} \alpha\left(T_{s}\right)
$$

$$
\beta(T)=\sum_{s=1}^{k} \beta\left(T_{s}\right)
$$

$$
\gamma_{i, j}(T)=\left\{\begin{array}{l}
\sum_{s=1}^{k} \gamma_{i, j}\left(T_{s}\right):(i, j) \neq(k, l), \\
\sum_{s=1}^{k} \gamma_{k, l}\left(T_{s}\right)+1:(i, j)=(k, l), \\
\quad i=1, \ldots, m, j=0, \ldots, i,(i, j) \neq(1,1) .
\end{array}\right.
$$

Hence (4.10) is true.

To complete the proof we need to demonstrate that $\beta(T)$ is the number of top vertices of $T$. This follows at once by induction from the composition rules and (4.12).

Corollary. $\beta(T)$ is the sum of coefficients in $\lambda_{T}$. In other words, letting $\lambda_{T}=$ $\lambda_{T}(q)$, it is true that $\beta_{T}=\lambda_{T}(1)$.

Proof. A straightforward inductive argument applied to (4.9).

We let

$$
\gamma(T):=\sum_{i=1}^{m} \sum_{\substack{j=0 \\(i, j) \neq(1,1)}}^{i} \gamma_{i, j}(T), \quad T \in \mathbb{T} .
$$

Proposition 9. The function $\alpha(T)$ can be represented in the form

$$
\alpha(T)=\prod_{r=1}^{\gamma(T)}\left(\lambda_{S_{r}(T)}-1\right),
$$

where $S_{1}(T), S_{2}(T), \ldots, S_{\gamma(T)}(T) \in \mathbb{T} \backslash\left\{T_{\circ}\right\}$.

Proof. It follows from (4.11) that $\alpha(T)$ can be factorized as a product of the form

$$
\alpha(T)=\prod_{r=1}^{\mu(T)}\left(\lambda_{S_{r}(T)}-1\right),
$$

where $\mu: \mathbb{T} \rightarrow \mathbb{Z}^{+}$obeys

$$
\mu\left(T_{\circ}\right)=1, \quad \mu(T)=\sum_{s=1}^{k} \mu\left(T_{s}\right)+1,
$$


whereby, like before, $T$ originates from $T_{1}, T_{2}, \ldots, T_{k}$ by the second composition rule. However, according to (4.12), (4.13) and the definition of $\gamma(T)$, the latter function obeys (4.14). Since $\gamma$ and $\mu$ obey the same recurrence relation and share an identical initial value, they necessarily coincide.

Table 1 (see next page) displays all distinct members of $\mathbb{T}$ of order $\leq 4$, subject to the assumption that no coefficients $a_{k, l}$ vanish. Several important features of our construction are illustrated by this table. Firstly, elements of $\mathbb{T}$ are multiply-rooted planar trees together with their functions $\alpha$ and $\gamma$ ! It is perfectly possible (and, indeed, likely) for the composition rules to produce identical members of $\mathbb{W}$ with different values of $\alpha$ and $\gamma$. Thus, letting

$$
\left.T_{1}:=\right\rfloor, \quad T_{2}:=\bullet, \quad T_{3}:=〕 \bullet,
$$

we obtain (subject to all the 'right' $a_{k, l}$ 's being nonzero)

$$
\begin{aligned}
& \longleftrightarrow=T_{1}-T_{0}-T_{0} \quad\left(\gamma(T)=2, \quad \alpha(T)=q^{2}-1\right) \\
& =T_{1}-T_{2} \quad\left(\gamma(T)=3, \quad \alpha(T)=q^{2}-1\right) \\
& =T_{3}-T_{0} \quad\left(\gamma(T)=3, \quad \alpha(T)=q\left(q^{2}-1\right)\right) .
\end{aligned}
$$

As long as the values of $\gamma$ and $\alpha$ are clear (or do not matter), we will continue to refer to members of $\mathbb{T}$ as 'trees' and employ the same notation as for elements of $\mathbb{W}$. However, we must bear in mind that composition attaches two 'labels' to each tree.

Secondly, the same tree, with identical values of $\gamma$ and $\alpha$, can be frequently composed in a number of ways. Thus

$$
T=\bullet \bullet=T_{0}-T_{3}=T_{4}-T_{0} \quad \text { where } \quad T_{4}:=\bullet \text { ?. }
$$

We choose $T_{4}$ such that $\gamma\left(T_{4}\right)=2$. In either case we obtain $\gamma(T)=3$, $\alpha(T)=q\left(q^{2}-1\right)$. In principle, we could have dealt with this by allocating multiplicities, but this is hardly necessary, since members of $\mathbb{T}$ are counted (in Proposition 10) indirectly, by employing composition rules.

Thirdly, an identical value of $\lambda_{T}$ may occur repeatedly in $\mathbb{T}$. For example (which requires a trivial extension of Table 1 ), letting $T_{5}:=\bullet \bullet \bullet$ and bearing in mind that $\gamma\left(T_{5}\right)$ is either 1 or 2 , we obtain inter alia the following $T \in \mathbb{T}$, all with $\lambda_{T}=3 q$ :

a. $a_{3,0} \neq 0$ :

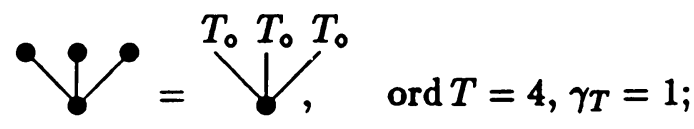

b. $a_{1,0}, a_{3,3} \neq 0$ :

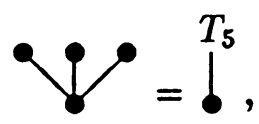

$$
\operatorname{ord} T=4, \gamma_{T}=2
$$


c. $a_{1,0}, a_{2,2} \neq 0$ :

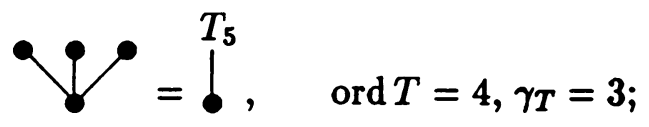

d. $a_{1,0}, a_{3,1} \neq 0$ :

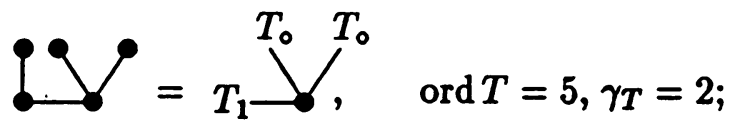

e. $a_{1,0}, a_{3,2} \neq 0$ :

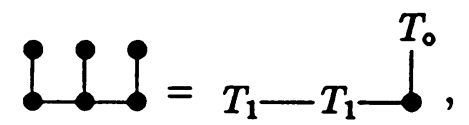
$\operatorname{ord} T=6, \gamma_{T}=3$;

f. $a_{1,0}, a_{3,3} \neq 0$ :<smiles>CCC(C)C=[Te][Al][Al]</smiles>
$\operatorname{ord} T=6, \gamma_{T}=4$

More trees such that $\lambda_{T}=3 q$ can be composed, and this is left to the reader.

Table 1. All distinct $T \in \mathbf{W}$, ord $T \leq 4, m \geq 4$, with their $\lambda_{T}, \alpha(T), \beta(T)$ and $\gamma(T)$ functions.

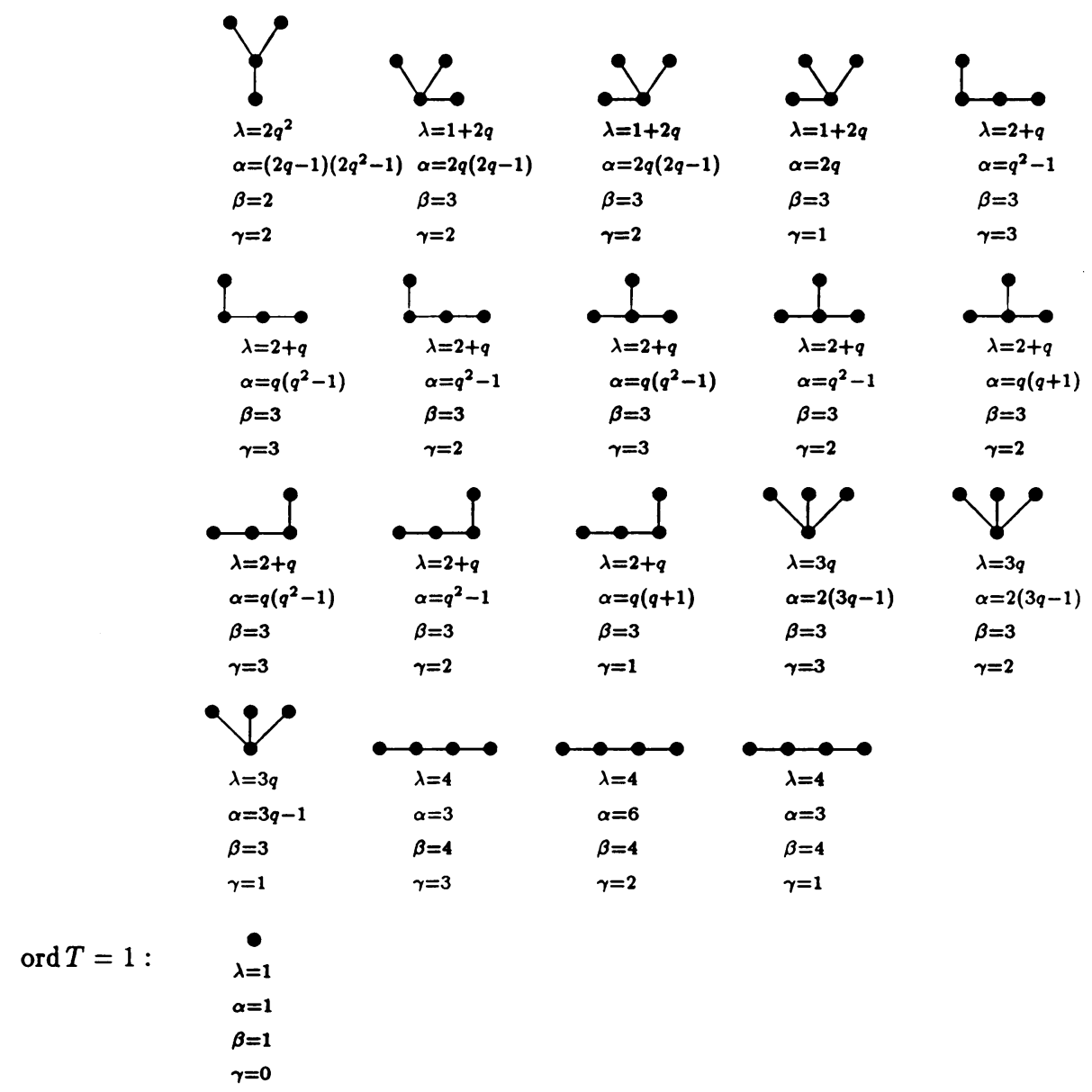


Table 1 (Continued)

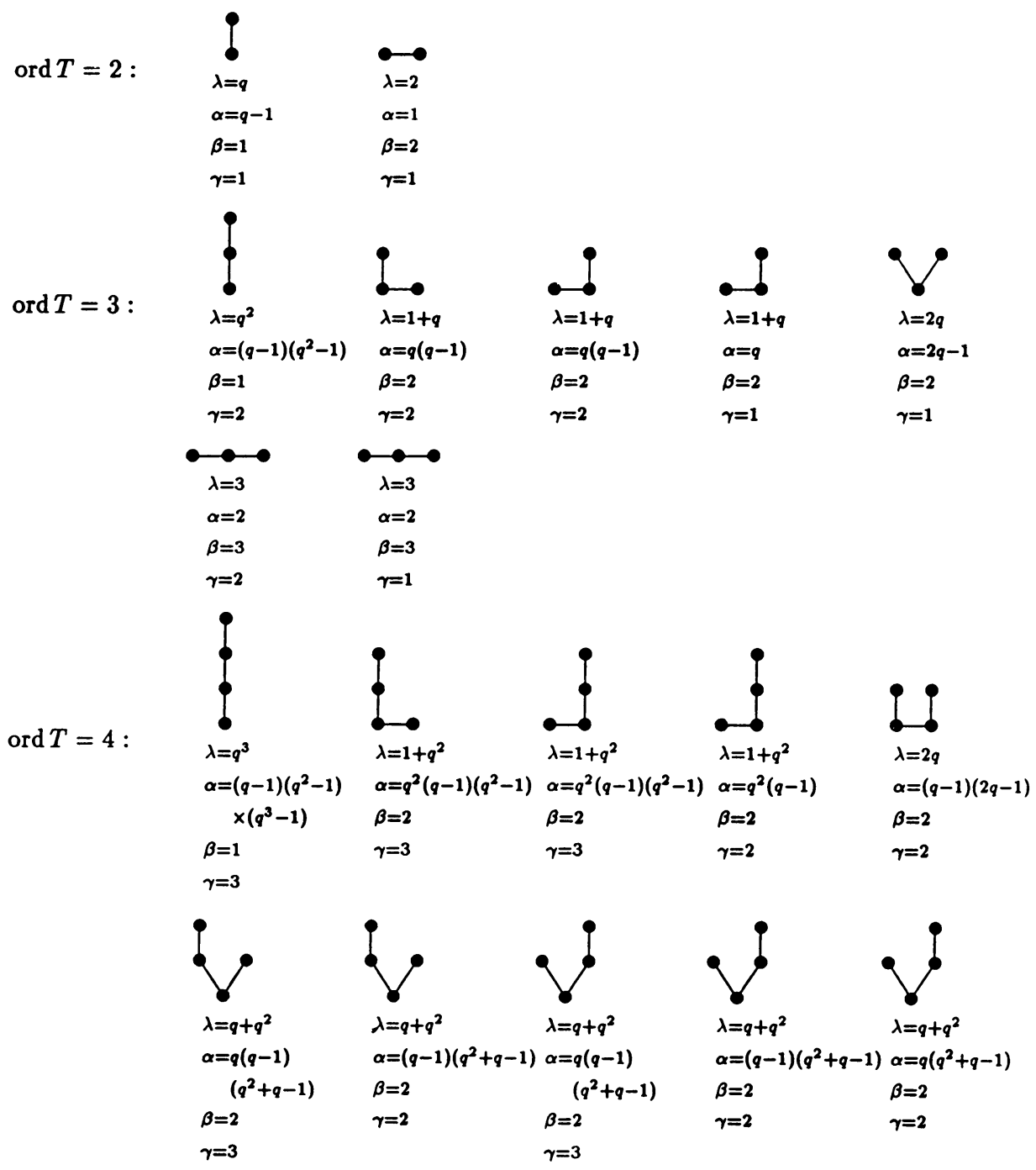

It should be obvious by now that an important distinction needs to be drawn between $\beta(T)$ on the one hand and $\gamma(T)$ and $\alpha(T)$ on the other. Whereas $\beta(T)$ is a structural property of $T \in \mathbb{W}$ and can be deduced directly from the tree (and, indeed, from $\left.\lambda_{T}\right), \gamma(T)$ and $\alpha(T)$ depend on the derivation of $T \in \mathbb{T}$ by a specific sequence of composition rules-indeed, an intuitive meaning of $\gamma(T)$ is as the number of composition that have been required to produce $T \in \mathbb{T}$.

Let us suppose that there exists $\eta=\eta(q)>0$ such that

$$
\left|\lambda_{T}-1\right| \geq \eta, \quad T \in \mathbb{T} \backslash\left\{T_{\circ}\right\} .
$$

Therefore, by Proposition 9,

We set

$$
\frac{1}{|\alpha(T)|} \leq \eta^{-\gamma(T)}, \quad T \in \mathbb{T} .
$$

$$
\sigma:=\max \left\{\left|\omega_{i, j}\right|: i=1,2, \ldots, m, j=0,1, \ldots, i,(i, j) \neq(1,1)\right\}
$$


and use (4.10) to deduce the inequality

$$
\left|d_{T}\right| \leq\left|d_{T_{\circ}}\right|^{\beta(T)}(\sigma / \eta)^{\gamma(T)} .
$$

Inequality (4.16) is important to our goal of bounding

$$
d^{*}:=\sum_{T \in \mathbb{T}}\left|d_{T}\right| \text {. }
$$

To that end we partition $\mathbb{T}$ into the sets

$$
\mathbb{B}_{n, s}:=\{T \in \mathbb{T}: \beta(T)=n, \gamma(T)=s\},
$$

where $n=1,2, \ldots$ and $s=0,1, \ldots$ Let $M_{n, s}$ be the number of elements in $\mathbb{B}_{n, s}$. Note that many $M_{n, s}$ 's equal zero, but this creates no problems whatsoever.

According to (4.16), we have

$$
\begin{aligned}
d^{*} & \leq \sum_{T \in \mathbb{T}}\left|d_{T_{\circ}}\right|^{\beta(T)}\left(\frac{\sigma}{\eta}\right)^{\gamma(T)} \\
& =\sum_{n=1}^{\infty} \sum_{s=0}^{\infty}\left|d_{T_{\circ}}\right|^{n}\left(\frac{\sigma}{\eta}\right)^{s}=\left|d_{T_{\circ}}\right| M\left(\left|d_{T_{\circ}}\right|, \sigma / \eta\right),
\end{aligned}
$$

where

$$
M(x, y):=\sum_{n=1}^{\infty} \sum_{s=0}^{\infty} M_{n, s} x^{n-1} y^{s}
$$

In order to take advantage of (4.17) in the context of the analysis of $\S 3$, we require $(\tilde{x}, \tilde{y})$, where $\tilde{x}=\left|d_{T_{\circ}}\right|, \tilde{y}:=\sigma / \eta$, to lie inside the domain of convergence of the Taylor series of $M$. Thus, our next step is to take a closer look at the bivariate function $M$. This will be done in several stages. In the next three propositions we assume that all the underlying sums absolutely converge.

Proposition 10. For every $n=1,2, \ldots$

(4.18) $M_{n, 0}=\delta_{n, 1}$,

$$
M_{n, s} \leq \sum_{k=1}^{m} k \sum_{\substack{i_{1}, \ldots, i_{k} \geq 1 \\ i_{1}+\cdots+i_{k}=n}} \sum_{\substack{j_{1}, \ldots, j_{k} \geq 0 \\ j_{1}+\cdots+j_{k}=s-1}} \prod_{r=1}^{k} M_{i_{r}, j_{r}}, \quad s=1,2, \ldots
$$

Proof. It is a straightforward consequence of (4.13) that there is just one $T \in \mathbb{T}$ such that $\gamma(T)=0$, namely $T_{\circ}$. Since $\beta\left(T_{\circ}\right)=1$, this proves (4.18).

To prove (4.19) we observe from (4.5), (4.12) and (4.13) that any $T \in$ $\mathbb{B}_{n, s}, s \geq 1$, 'originates' from some $T_{1}, T_{2}, \ldots, T_{k}$, where $T_{r} \in \mathbb{B}_{i_{r}, j_{r}}$, $r=1,2, \ldots, k$, and $\sum_{r=1}^{k} i_{r}=n, \sum_{r=1}^{k} j_{r}=s-1$. Moreover, by allowing $k$ and $l$ to range in $\{1,2, \ldots, m\}$ and $\{0,1, \ldots, k\}$ respectively, $(k, l) \neq(1,1)$, we can recover in this manner all $T \in \mathbb{B}_{n, s}$. This proves the required inequality.

Let us define recursively for $n=1,2, \ldots$

$$
M_{n, 0}^{*}:=\delta_{n, 1} \text {, }
$$

$$
M_{n, s}^{*}:=\sum_{k=1}^{m} k \sum_{\substack{i_{1}, \ldots, i_{k} \geq 1 \\ i_{1}+\cdots+i_{k}=n}} \sum_{\substack{j_{1}, \ldots, j_{k} \geq 0 \\ j_{1}+\cdots+j_{k}=s-1}} \prod_{r=1}^{k} M_{i_{r}, j_{r}}^{*}, \quad s=1,2, \ldots
$$


We may deduce at once from (4.20) and Proposition 10 that

$$
M(x, y) \leq M^{*}(x, y):=\sum_{n=1}^{\infty} \sum_{l=0}^{\infty} M_{n, s}^{*} x^{n-1} y^{s}, \quad x, y \geq 0
$$

Our next result is a technical lemma that, in all likelihood, is already well known. However, as its proof is absolutely straightforward, we include it for completeness.

Proposition 11. Given integers $k \geq 1$ and $r \geq 0$ and sequences $\left\{\alpha_{l, s}: l=\right.$ $1,2, \ldots, k, s=r, r+1, \ldots\}$ and assuming that all underlying sums absolutely converge, it is true that

$$
\sum_{s=r}^{\infty} \sum_{\substack{j_{1}, \ldots, j_{k} \geq r \\ j_{1}+\cdots+j_{k}=s}} \prod_{l=1}^{k} \alpha_{l, j_{l}}=\prod_{l=1}^{k}\left(\sum_{s=r}^{\infty} \alpha_{l, s}\right) .
$$

Proof. The identity (4.22) is trivial for $k=1$, whereas for $k \geq 2$ we have, after changing the order of summation,

$$
\begin{aligned}
\sum_{s=r}^{\infty} & \sum_{\substack{j_{1}, \ldots, j_{k} \geq r \\
j_{1}+\cdots+j_{k}=s}} \prod_{l=1}^{k} \alpha_{l, j_{l}} \\
& =\sum_{s=r}^{\infty} \sum_{j_{1}=r}^{s-(k-1) r} \sum_{j_{2}=r}^{s-(k-2) r-j_{1}} \ldots \sum_{j_{k-1}=r}^{s-r-\sum_{i=1}^{k-2} j_{i}}\left(\prod_{l=1}^{k-1} \alpha_{l, j_{l}}\right) \alpha_{k, s-\sum_{i=1}^{k-1} j_{i}} \\
= & \sum_{j_{1}=r}^{\infty} \sum_{j_{2}=r}^{\infty} \cdots \sum_{j_{k-1}=r}^{\infty} \sum_{s=r+\sum_{i=1}^{k-1} j_{i}}^{\infty-1}\left(\prod_{l=1}^{\infty} \alpha_{l, j_{l}}\right) \alpha_{k, s-\sum_{i=1}^{k-1} j_{i}} \\
= & \prod_{l=1}^{k}\left(\sum_{s=r}^{\infty} \alpha_{l, s}\right) .
\end{aligned}
$$

Proposition 12. The functions

$$
\mathscr{M}_{s}^{*}(x):=\sum_{n=1}^{\infty} M_{n, s}^{*} x^{n-1}, \quad s \in \mathbb{Z}^{+},
$$

obey the recurrence relation

$$
\begin{aligned}
& \mathscr{M}_{0}^{*}(x) \equiv 1, \\
& \mathscr{M}_{s}^{*}(x)=\sum_{k=1}^{m} k x^{k-1} \sum_{\substack{j_{1}, \ldots, j_{k} \geq 0 \\
j_{1}+\cdots+j_{k}=s-1}} \prod_{l=1}^{k} \mathscr{M}_{j_{l}}^{*}(x), \quad s=1,2, \ldots
\end{aligned}
$$

Proof. Whereas (4.23) follows at once from (4.18), we prove (4.24) by multiplying (4.20) by $x^{n-1}$ and summing up for $n=1,2, \ldots$. This gives 


$$
\begin{aligned}
\mathscr{M}_{s}^{*}(x) & =\sum_{n=1}^{\infty} x^{n-1} \sum_{k=1}^{m} k \sum_{\substack{i_{1}, \ldots, i_{k} \geq 1 \\
i_{1}+\cdots+i_{k}=n}} \sum_{j_{1}+\cdots, j_{k}=s-1} \prod_{j_{1}, \ldots, j_{k} \geq 0}^{k} M_{i_{l}, j_{l}}^{*} \\
& =\sum_{k=1}^{m} x^{k-1} k \sum_{\substack{j_{1}, \ldots, j_{k} \geq 0 \\
j_{1}+\cdots+j_{k}=s-1}} \sum_{n=1}^{\infty} \sum_{\substack{i_{1}, \ldots, i_{k} \geq 1 \\
i_{1}+\cdots+i_{k}=n}} \prod_{l=1}^{k} M_{i_{l}, j_{l}}^{*} x^{i_{l}-1} .
\end{aligned}
$$

We now use Proposition 11 (with $r=1$ and $\alpha_{l, j}=M_{i_{l}, j}^{*} x^{i_{l}-1}$ ) to argue that the recurrence $(4.24)$ holds.

Proposition 13. Given that $x, y \geq 0$ are sufficiently small, the function $M^{*}(x, y)$ is the unique zero of the polynomial

$$
\begin{aligned}
\theta_{x, y}(t) & =1-t+y \sum_{k=1}^{m} k x^{k-1} t^{k} \\
& =1-t+y t \frac{1-(m+1)(x t)^{m}+m(x t)^{m+1}}{(1-x t)^{2}}, \quad x, y \in \mathbb{R}^{+},
\end{aligned}
$$

that obeys $M^{*}(0,0)=1$.

Proof. Since $M^{*}(x, y)=\sum_{s=0}^{\infty} \mathscr{M}_{s}^{*}(x) y^{s}$, we multiply (4.24) by $y^{s}$, sum up for $s=1,2, \ldots$ and add $(4.23)$. This results in

$$
\begin{aligned}
M^{*}(x, y) & =1+\sum_{s=1}^{\infty} \sum_{k=1}^{m} k x^{k-1} y^{s} \sum_{\substack{j_{1}, \ldots, j_{k} \geq 0 \\
j_{1}+\cdots+j_{k}=s-1}} \prod_{l=1}^{k} \mathscr{M}_{j_{l}}^{*}(x) \\
& =1+y \sum_{k=1}^{m} k x^{k-1} \sum_{s=1}^{\infty} \sum_{\substack{j_{1}, \ldots, j_{k} \geq 0 \\
j_{1}+\cdots+j_{k}=s}} \prod_{l=1}^{k} \mathscr{M}_{j_{l}}^{*}(x) y^{j_{l}}
\end{aligned}
$$

We next employ Proposition 11 with $r=0$ and $\alpha_{l, j}=\mathscr{M}_{l}^{*}(x) y^{l}$ to argue that

$$
M^{*}(x, y)=1+y \sum_{k=1}^{m} k x^{k-1}\left(M^{*}(x, y)\right)^{k} .
$$

Therefore $\theta_{x, y}\left(M^{*}(x, y)\right)=0$.

As $x, y \rightarrow 0, m-1$ zeros of $\theta_{x, y}$ travel to infinity and one to 1 . Since $M^{*}(0,0)=1$, it is the latter that is identified with $M^{*}$. This identification is valid sufficiently near to $(x, y)=(0,0)$, since $\theta_{0,0}^{\prime}(1) \neq 0$.

Note that, because of (4.21), existence of a convergent Taylor expansion of $M^{*}$ at $(x, y) \in \mathbb{R}^{+} \times \mathbb{R}^{+}$implies that this is also the case with $M$. The latter in turn proves, by virtue of $(4.18)$, that the Dirichlet series converges.

Of course, we are interested in $(x, y) \in \mathbb{R}^{+} \times \mathbb{R}^{+}$away from $(0,0)$. It follows from standard theory of analytic functions that the bivariate Taylor series for $M$ converges (to the correct zero of $\left.\theta_{x, y}\right)$ for all $(x, y) \in \mathscr{G}_{m}$, where the set $\mathscr{G}_{m}$ is the intersection of $\mathbb{R}^{+} \times \mathbb{R}^{+}$with the natural projection of the largest connected portion of the sheet of the covering Riemann surface of $\theta_{x, y}$ that crosses $(0,0)$ at $t=1$ and excludes branch points and branch cuts. 
In other words, $\mathscr{G}_{m}$ is the largest subset of the nonnegative quadrant such that for every $(x, y)$ the solution $t_{x, y}$ of $\theta_{x, y}\left(t_{x, y}\right)=0$ possesses a convergent Taylor expansion and there exists a unique analytic continuation from $t_{0,0}=1$ to $t_{x, y}$.

A long but straightforward method to produce $\mathscr{G}_{m}$ is as follows: the boundary of the set is either one of the lines $x=0$ or $y=0$, or it must be a branch point. ${ }^{3}$ To determine the latter, apply the Euclidean algorithm to the polynomials

$$
\begin{aligned}
& \theta_{x, y}(t)=1-t+y \sum_{k=1}^{m} k x^{k-1} t^{k} \\
& \theta_{x, y}^{\prime}(t)=-1+y \sum_{k=0}^{m-1}(k+1)^{2} x^{k} t^{k}
\end{aligned}
$$

This results in a high-degree bivariate polynomial, $G_{m}(x, y)$, say. All points of $(x, y) \in \mathbb{C}^{2}$ such that $G_{m}(x, y)=0$ are branch points of $\theta_{x, y}$. This defines a family of curves. The set $\mathscr{G}_{m}$ is the largest connected portion of $\mathbb{R}^{+} \times \mathbb{R}^{+}$that contains $(0,0)$ and excludes all solutions of $G_{m}(x, y)=0$.

In the simplest nontrivial case $m=2$ we have

$$
G_{2}(x, y)=(1-y)^{2}-8 x y
$$

therefore

$$
\mathscr{G}_{2}=\left\{(x, y): 0 \leq x y<\frac{1}{8}(1-y)^{2}, 0 \leq y<1\right\}
$$

Likewise, a slightly more substantial calculation affirms that

$$
G_{3}(x, y)=8 y^{3}-76 x y^{2}+243 x^{2}-28 y^{2}+108 x y+32 y-12
$$

and

$$
\mathscr{G}_{3}=\left\{(x, y): 0 \leq x<\frac{2}{243}\left(19 y-27+\sqrt{\frac{(9-5 y)^{3}}{y}}\right), 0 \leq y<1\right\} .
$$

Larger values of $m$ require symbolic manipulation. Thus, for example,

$$
\begin{aligned}
G_{4}(x, y)= & 3031040 x^{3} y^{2}-1706995 x^{2} y^{3}+418100 x y^{4}-37000 y^{5} \\
& +10911744 x 3 y-4440222 x^{2} y^{2}+410700 x y^{3}+40700 y^{4} \\
& +6137856 x^{2} y-3127536 x y^{2}+309320 y^{3}+2925072 x y \\
& -880452 y^{2}+855144 y-287712
\end{aligned}
$$

Since the Taylor coefficients of $M^{*}$ are all nonnegative, the domain of convergence decreases with $m$. Letting $m \rightarrow \infty$ in the definition of $\theta_{x, y}$ we thus obtain a lower bound on the set $\mathscr{G}_{m}$. In other words,

$$
\mathscr{G}_{\infty} \subseteq \cdots \subseteq \mathscr{G}_{m+1} \subseteq \mathscr{G}_{m} \subseteq \cdots \text {. }
$$

To evaluate $\mathscr{G}_{\infty}$ we note that

$$
\lim _{m \rightarrow \infty} \theta_{x, y}(t)=1-t+\frac{y t}{(1-x t)^{2}}, \quad|x t|<1,
$$

3 The phrase 'branch point' is a misnomer in the present context, since these points are not isolated and they lie along 'branch curves'. 

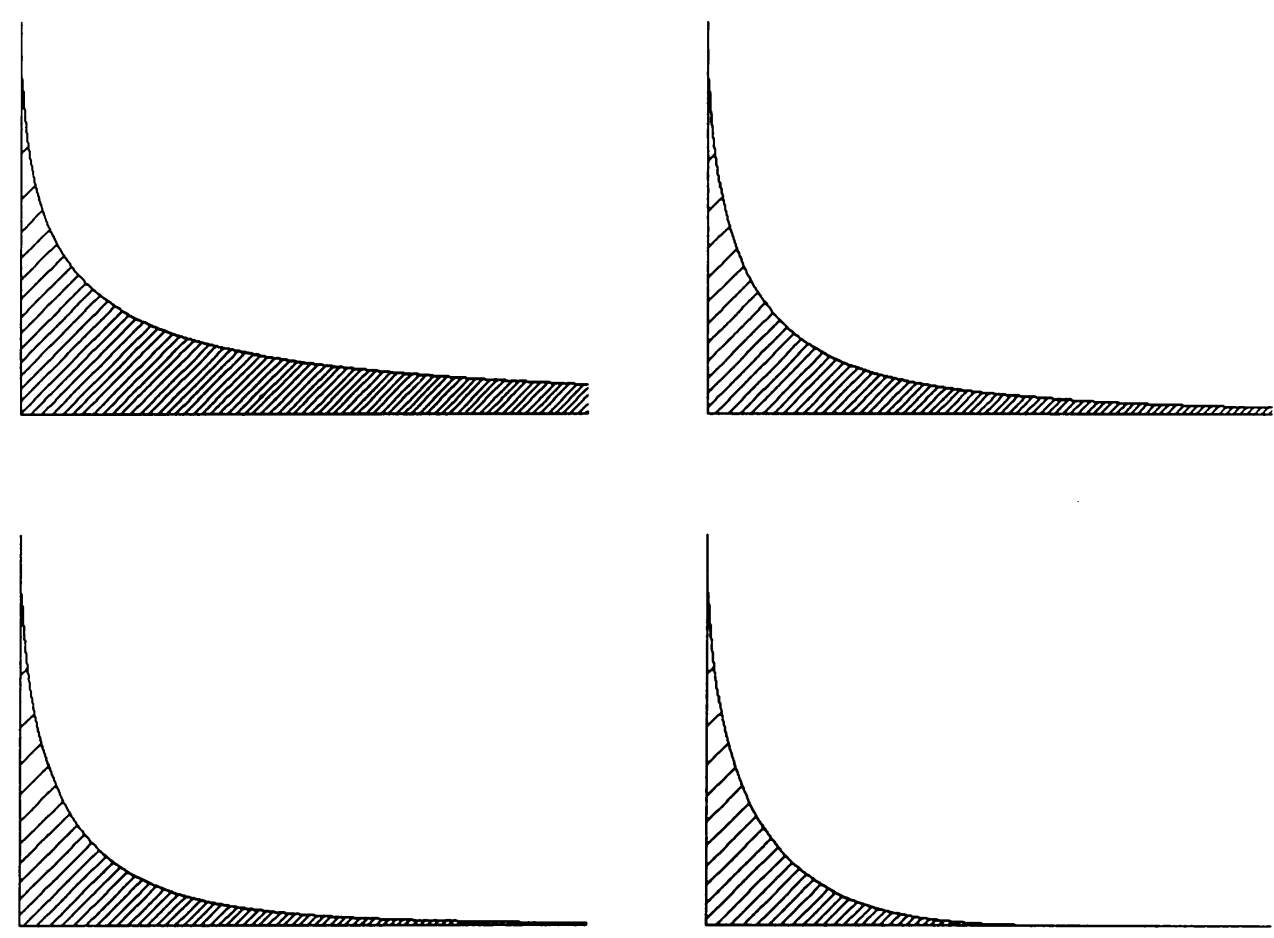

FIGURE 2. The sets $\mathscr{G}_{m}$ for $m=2,3,4, \infty$

therefore

$$
G_{\infty}(x, y)=4 x^{3}-x^{2} y-12 x^{2}+20 x y-4 y^{2}+12 x+8 y-4 .
$$

Simple calculation affirms that

$$
\mathscr{G}_{\infty}=\left\{(x, y): 0 \leq x \leq 1,0 \leq y \leq \frac{1}{8}\left(8+20 x-x^{2}-\sqrt{x(x+8)^{3}}\right)\right\} .
$$

Figure 2 displays the sets $\mathscr{G}_{m}$ for $m=2,3,4$ and $m=\infty$, the scale being $0 \leq x \leq \frac{3}{2}, 0 \leq y \leq 1$. All these sets are consistent with the assertion (which, in its generality, is a tentative conjecture) that the boundary of $\mathscr{G}_{m}$ in $\{(x, y): x, y>0\}$ is a strongly concave, strictly monotonically decreasing curve, rapidly tending from above to the corresponding portion of $\partial \mathscr{G}_{\infty}$.

For all $m \geq 2$ and small $x>0$ substitution into (4.25) and (4.26) yields

$$
x=\frac{(1-y)^{2}}{8 y}+\mathscr{O}\left(x^{2}\right), \quad 0<y<1,
$$

as the boundary of $\mathscr{G}_{m}$. Thus, asymptotically for small $x, \mathscr{G}_{m}$ is similar to $\mathscr{G}_{2}$ and, more importantly, for every $y \in(0,1)$ there exists $\vartheta_{m, y}>0$ such that $(x, y) \in \mathscr{G}_{m}$ for all $x \in\left[0, \vartheta_{m, y}\right)$.

Likewise, asymptotics, consistent with our conjecture, are available for $x \gg$ 1. We assume that $y \sim \chi x^{-m+1}$ for some $\chi \in \mathbb{R}$. Substitution into (4.25) and (4.26) yields $1-t+m \chi t^{m}=o(1)$ and $-1+m^{2} \chi t^{m-1}=o(1)$ respectively. It is now easy to execute the Euclidean algorithm explicitly and derive $\chi=$ 
$(m-1)^{m-1} / m^{m+1}$. Thus, for $x \gg 1$ the 'upper' boundary of $\mathscr{G}_{m}$ is

$$
x^{m-1} y \approx \frac{(m-1)^{m-1}}{m^{m+1}}
$$

As $m \rightarrow \infty$, this portion of $\mathscr{G}_{m}$ shrinks, ultimately disappearing in the bounded set $\mathscr{G}_{\infty}$.

As long as $(\tilde{x}, \tilde{y}) \in \mathscr{G}_{m}$, we can be assured that the Taylor series for $M$ converges, hence $d^{*}$ can be bounded by (4.17). This, together with the theory of $\S 3$, provides useful information on the asymptotic behaviour of (4.4).

Theorem 14. Suppose that there exists $\eta>0$ such that $\left|\lambda_{T}-1\right| \geq \eta, T \neq T_{\circ}$, and that

$$
\left(\left|d T_{\circ}\right|, \sigma / \eta\right) \in \mathscr{G}_{m}
$$

Then a solution of the equation (4.4) can be expanded into convergent Dirichlet series for all $t \in\left[0, t^{*}\right)$ for some $t^{*}>0$. Moreover, if $\operatorname{Re} b \leq 0$ then $t^{*}=\infty$ and

a. If $\operatorname{Re} b<0$ then the solution is asymptotically stable;

b. If $\operatorname{Re} b=0, b \neq 0$, then the solution is almost periodic.

Proof. An immediate consequence of the theory of $\S 3$.

Note a major drawback in the last theorem, namely that, unlike a Taylor expansion, a Dirichlet expansion is, in general, difficult to match with an initial value. Thus, to generate a Dirichlet expansion we need to choose $d_{T_{0}} \in \mathbb{C}$, and this, in tandem with the recurrence (4.5), leads to $\Lambda$ and to Dirichlet coefficients $\mathscr{D}:=\left\{d_{T}\right\}_{T \in \mathbb{T}}$. Finally, provided that $\mathscr{D}$ is known, we can recover the initial value $y(0)=\sum_{T \in \mathbb{T}} d_{T}$. Hence, not much can be deduced from Theorem 14 in the event when $y(0)$ is given a priori. ${ }^{4}$ However, the recurrence (4.5) being homogeneous, $d_{T_{\circ}}=0$ implies $y(0)=0$ and, by continuity, small $\left|d_{T_{0}}\right|$ corresponds to small $|y(0)|$. Suppose that $\sigma<\eta$, hence $\tilde{y} \in(0,1)$. It has been already proved that for every $m \geq 2$ and every $y \in(0,1)$ there exists $\vartheta_{m, y}>0$ such that $\left(\left[0, \vartheta_{m, y}\right), y\right) \subset \mathscr{G}_{m}$. In other words, provided that $|y(0)|$ is sufficiently small, (4.27) holds.

Theorem 15. Suppose that $\operatorname{Re} b \leq 0$, that there exists $\eta>0$ such that $\left|\lambda_{T}-1\right| \geq$ $\eta, T \neq T_{\circ}$ and that

$$
\max \left\{\left|a_{i, j}\right|: i=1,2, \ldots, m, j=0,1, \ldots, i,(i, j) \neq(1,1)\right\}<\eta|b|
$$

Then there exists $y^{*}>0$ such that for all $|y(0)| \leq y^{*}$ the solution of (4.4) is asymptotically stable (if $\operatorname{Re} b<0$ ) or almost periodic (if $\operatorname{Re} b=0, b \neq 0$ ). In particular, if $\operatorname{Re} b<0$ then 0 is an attractive fixed point of (4.4). [4].

${ }^{4}$ The linear case $m=1$ is an exception, since then the map $d_{T_{\circ}} \rightarrow y(0)$ is linear and invertible 
The existence of $\eta>0$ is critical to the last two theorems. It will be debated further in the next section where we demonstrate that, unfortunately, the assumption requires a restriction of the range of $q>0$.

\section{ON THE EXISTENCE OF $\eta>0$}

We commence this section by considering the equation

$$
y^{\prime}(t)=b y(t)+\sum_{k=1}^{m} a_{k, 0} y^{k}(q t), \quad y(0)=y_{0} .
$$

The set $\mathbb{T}$ can be now derived in an explicit manner, since each $T \in \mathbb{T}$, ord $T \geq$ 2 , must be obtained by rooting a sum of $l \in\{1,2, \ldots, m\}$ members of $\mathbb{T}$, i.e.

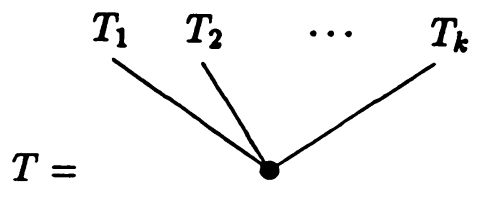

Consequently,

$$
\lambda_{T}=q \sum_{s=1}^{k} \lambda_{T_{s}} .
$$

The equation (5.1) is singled out by the fact that, unless $l=0$ in (4.8), the underlying $d_{T}$ must vanish. It is straightforward to deduce that $\mathbb{T}$ encompasses all connected, $m$-ary rooted planar trees. Table 2 displays all members of $\mathbb{T}$ of order $\leq 5$ in the case $m=3$

Lemma 16. Suppose that $a_{k, l}=0$ for all $k \geq 1$ and $l \geq 2$, hence that (4.4) reduces to $(5.1)$. Then

a. If $0<q<\frac{1}{m}$ then $0<\lambda_{T}<m q<1$ for all $T \in \mathbb{T} \backslash\left\{T_{\circ}\right\}$;

b. If $\frac{1}{m} \leq q \leq 1$ then 1 is an accumulation point of the set $\Lambda$;

c. If $1<q$ then $\lambda_{T}>q>1$ for all $T \in \mathbb{T} \backslash\{0\}$.

Proof. We already know that $\mathbb{T}$ is in the present case the set of all $m$-ary rooted planar trees.

Let firstly $q \in\left(0, \frac{1}{m}\right]$. We prove that $\lambda_{T} \leq m q$ by induction on ord $T \geq 2$, noting that $\lambda_{T}=q \leq m q$ for the unique $T \in \mathbb{T}$ of order 2 . Let the statement be true for all $T \in \mathbb{T}, 2 \leq \operatorname{ord} T \leq n-1$ and suppose that ord $T=n$ and that $T$ is constituted by rooting the sum of $T_{1}, T_{2}, \ldots, T_{k}$ as in (5.2). Since ord $T_{s} \leq n-1, s=1, \ldots, k$, it follows by induction and (5.3) that

$$
\lambda_{T} \leq q\left\{\sum_{\operatorname{ord} T_{i} \geq 2} m q+\sum_{\operatorname{ord} T_{i}=1} 1\right\} \leq m q .
$$

In the case of $q \in\left[\frac{1}{m}, 1\right]$ we commence by proving that for every $s \in \mathbb{Z}^{+}$ and $r \in\left\{1,2, \ldots, m^{s}\right\}$ there exists $T \in \mathbb{T}$ such that $\lambda_{T}=r q^{s}$. This will 
Table 2. All $T \in \mathbb{T}$ with $m=3$ and ord $T \leq 5$

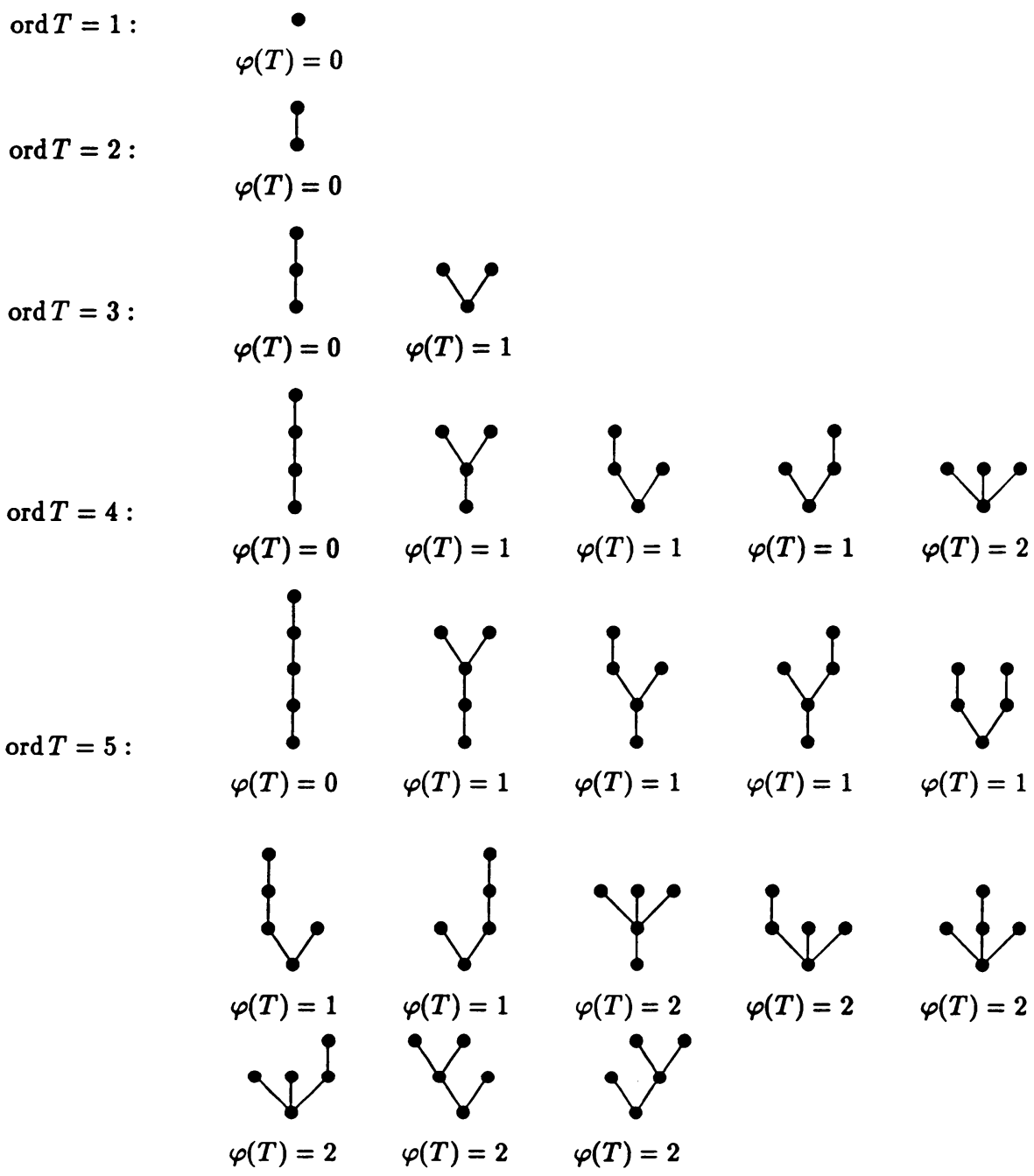

be done by induction on $s$, noting that the statement is true for $s=0$ since $\lambda_{T_{\circ}}=1$. Assume the validity of the statement for $s-1$, hence for every $i=1,2, \ldots, m^{s-1}$ there exists $T_{i} \in \mathbb{T}$ such that $\lambda_{T_{i}}=i q^{s-1}$. We form a new tree $T$ by appending a root to $T_{i_{1}}, T_{i_{2}}, \ldots, T_{i_{m}^{\prime}}$ in a manner identical to (5.2). Here $m^{\prime} \in\{1,2, \ldots, m\}$ and $1 \leq i_{1}<i_{2}<\cdots<i_{m^{\prime}} \leq m^{s-1}$. Therefore $T \in \mathbb{T}$ and, by (5.3),

$$
\lambda_{T}=q^{s} \sum_{j=1}^{m^{\prime}} i_{j} .
$$

It is straightforward to verify that

$$
\bigoplus_{m \text { times }}\left\{0,1, \ldots, m^{s-1}\right\}=\left\{0,1, \ldots, m^{s}\right\} .
$$


Consequently

$$
\left\{\sum_{j=1}^{m^{\prime}} i_{j}: 1 \leq i_{1}<i_{2}<\cdots<i_{m^{\prime}} \leq m^{s-1}, m^{\prime} \leq m\right\}=\left\{1,2, \ldots, m^{s}\right\}
$$

and $T$ such that $\lambda_{T}=r q^{s}$ exists for all $r=1,2, \ldots, m^{s}$.

Since $m q \geq 1$ and $q \leq 1$, there exists for every $s \geq 1$ a number $r_{s} \in$ $\left\{1,2, \ldots, m^{s}-1\right\}$ such that $1 \in\left(r_{s} q^{s},\left(r_{s}+1\right) q^{s}\right]$. Thus, for every such $s$ there exists a tree $T_{s}^{*} \in \mathbb{T}$ such that $\left|\lambda_{T_{s}^{*}}-1\right| \leq \frac{1}{2} q^{s}$ and 1 is an accumulation point of the set $\left\{\lambda_{T_{s}^{*}}: s=1,2, \ldots\right\} \subseteq \mathbb{T}$.

Finally, let $q>1$. To prove that $\lambda_{T} \geq q$ when ord $T \geq 2$ it is enough to note that, by (5.3), the polynomial $\lambda_{T}$ is a multiple of $q$ with integer nonnegative coefficients, not all of which may vanish. This completes the proof of the lemma.

Corollary. Expanding (5.1) into Dirichlet series, we may choose $\eta=1-m q$ for $q<\frac{1}{m}$ and $\eta=q-1$ for $q>1$.

The functions $\beta(T)$ and $\gamma(T)$ can be evaluated explicitly for (5.1). Let the fertility frt $v$ of a vertex $v \in T$, where $T \in \mathbb{T}$, be defined as the number of its children. We set

$$
\varphi(T):=\sum_{v \text { is a vertex of } T}(\text { frt } v-1)_{+}, \quad T \in \mathbb{T} .
$$

Proposition 17. For every $T \in \mathbb{T} \beta(T)=\varphi(T)+1$ and $\gamma(T)=\operatorname{ord} T-\varphi(T)-$ 1 .

The proof follows directly and in a straightforward manner from composition rules in $\S 4$ and is left for the reader.

Next we consider the equation (4.4) under the assumption that there exist $1 \leq k \leq m, 2 \leq l \leq m$ and $1 \leq p \leq l$ such that $a_{k, 0}, a_{l, p} \neq 0$. This is an almost complementary situation to the case (5.1).

According to (4.8), we have
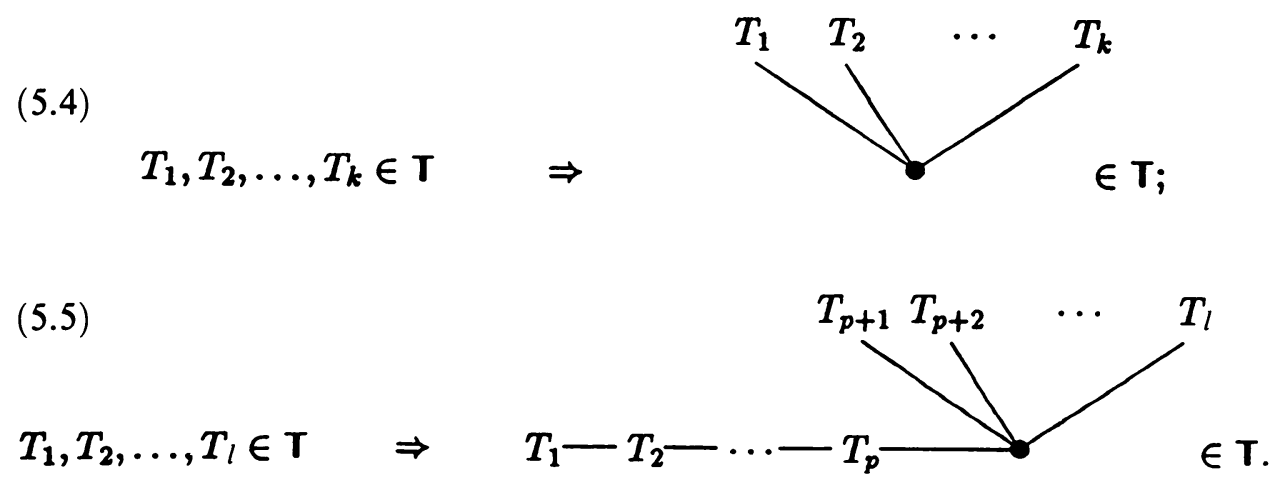

Lemma 18. Subject to the aforementioned conditions and stipulating that $q \leq \frac{1}{k}$, necessarily either 1 is an accumulation point of $\Lambda$ or there exists $T \in \mathbb{T} \backslash\left\{T_{\circ}\right\}$ such that $\lambda_{T}=1$.

Proof. For every $\varepsilon>0$ we use the rules (5.4) and (5.5) recursively to construct $\Lambda_{\varepsilon} \subset \Lambda$ that contains an element in an $\varepsilon$-neighbourhood of 1 . 
Let $r$ and $s$ in $\mathbb{Z}^{+}$. We set $T^{(0)}:=T_{\circ}$ and use (5.5) to generate

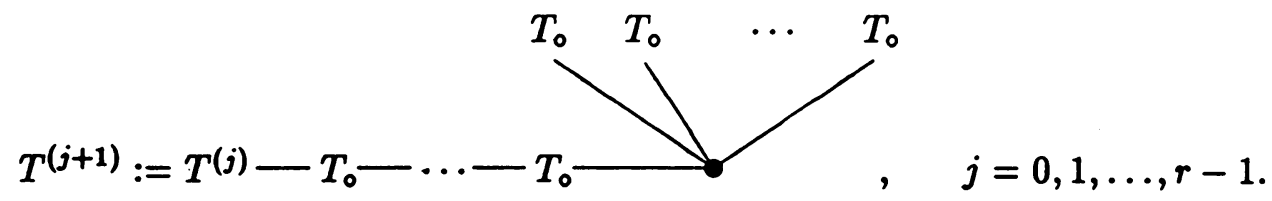

Since $\lambda_{T^{(0)}}=1$ and

$$
\lambda_{T^{(j+1)}}=\lambda_{T^{(j)}}+(p-1)+(l-p) q, \quad j=0,1, \ldots, r-1,
$$

it follows that

$$
\lambda_{T^{(j)}}=((p-1)+(l-p) q) j+1, \quad j=0,1, \ldots, r .
$$

Next we use (5.4) $s$ times, letting

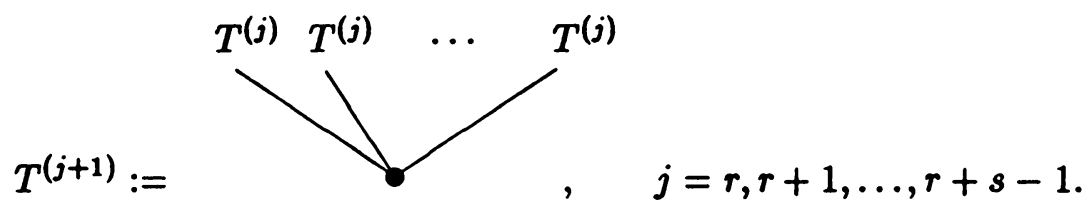

Since

$$
\lambda_{T^{(j+1)}}=k q \lambda_{T^{(j)}}, \quad j=r, r+1, \ldots, r+s-1,
$$

it follows that

$$
\lambda_{T^{(r+s)}}=(k q)^{s}(((p-1)+(l-p) q) r+1) .
$$

Let $T_{r, s}:=T^{(r+s)}, r, s \in \mathbb{Z}^{+}$and set $\Lambda_{s}:=\left\{\lambda_{T_{r, s}}: r \in \mathbb{Z}^{+}\right\}, s \in \mathbb{Z}^{+}$. Thus, if $k q=1$ then $\lambda_{T_{0, s}}=1, T_{0, s} \neq T_{\circ}$ for $s \geq 1$ and the lemma is true. Otherwise, the assumption $k q<1$ implies that $\lambda_{T_{0, s}}<1$, whereas, by (5.6), $\lambda_{T_{r, s}}>1$ for sufficiently large $r$. Moreover, the elements of $\Lambda_{s}$, ordered by $r$, form a monotonically increasing equidistant sequence,

$$
\lambda_{T_{r+1, s}}-\lambda_{T_{r, s}}=(k q)^{s}((p-1)+(l-p) q)=c_{s}>0,
$$

say. Since $k q<1$, sufficiently large $s$ makes $c_{s}$ as small as required. Thus, for every $\varepsilon>0$ there exists $s_{\varepsilon}$ such that for all $s \geq s_{\varepsilon}$ there is a member of $\Lambda_{s} \subset \Lambda$ in an $\varepsilon$-neighbourhood of 1 .

The import of Lemmas 16 and 18 is that Dirichlet expansions frequently fail when $q \in(0,1)$. Thus, in Lemma 18 it is only natural to choose $k \geq 1$ as the least index such that $a_{k, 0} \neq 0$. For example, if $a_{1,0} \neq 0$ then Dirichlet series fail for all $q \in(0,1) .{ }^{5}$ The disappointment is somewhat obviated, since it is easy to prove that Dirichlet expansions exist in the advanced case $q>1$. This has been already demonstrated for a special case in Lemma 16, but a more general result is easy to derive.

${ }^{5}$ Of course, the last statement requires $m \geq 2$. If $m=1$ and the equation is linear then Dirichlet series exists for every $q \in(0,1)[4]$. 


\section{Lemma 19.}

a. $q>1$ implies that $\lambda_{T} \geq \min \{2, q\}$ for all $T \in \mathbb{T} \backslash\left\{T_{\circ}\right\}$.

b. If $q \geq 2$ then $\lambda_{T} \geq$ ord $T$ for all $T \in \mathbb{T}$.

c. If $a_{k, k} \neq 0$ for some $k \in\{2,3, \ldots, m\}$ then for every $n \geq 1$ such that $(n-1)$ is divisible by $(k-1)$ there exists $T \in \mathbb{T}$ such that $\lambda_{T}=\operatorname{ord} T=n$.

Proof. We prove the first statement by induction on ord $T \geq 2$. Table 1 affirms that it is true for ord $T=2$. Let us suppose that $T \in \mathbb{T}$ has been derived from $T_{1}, T_{2}, \ldots, T_{k}$ via (4.8). Hence

$$
\lambda_{T}=\sum_{i=1}^{l} \lambda_{T_{i}}+q \sum_{i=l+1}^{k} \lambda_{T_{i}} .
$$

Note that $\operatorname{ord} T_{i}<\operatorname{ord} T$ for $i=1,2, \ldots, k$. Moreover, $\lambda_{T} \geq 1$ for all $T \in \mathbb{T}$, since $q>1$ and $\lambda_{T} \not \equiv 0$ is a polynomial with nonnegative integer coefficients in $q$. Therefore, $l \in\{1,2, \ldots, k-1\}$ and (5.7) imply that

$$
\lambda_{T} \geq q \min _{l+1 \leq i \leq k} \lambda_{T_{i}} \geq q \text {. }
$$

On the other hand, in the case $l=k$, there are two possibilities. Firstly, if $T_{i} \equiv T_{\circ}, i=1,2, \ldots, k$, then (5.7) gives $\lambda_{T}=k \lambda_{T_{\circ}}=k \geq 2$, whereas if $T_{j} \neq T_{\circ}$ for some $j \in\{1,2, \ldots, k\}$ then we use (5.7) and the induction assumption to argue that

$$
\lambda_{T} \geq \lambda_{T_{j}} \geq \min \{2, q\} .
$$

This completes the proof of our first assertion.

To prove that $\lambda_{T} \geq$ ord $T$ for $q \geq 2$ we again use (5.7) and induction on ord $T$. The statement is true for ord $T=1$, since $\lambda_{T_{\circ}}=1=$ ord $T_{\circ}$. Otherwise $T$ must have been derived from some $T_{1}, \ldots, T_{k} \in \mathbb{T}$ as in (4.8). Hence, by (5.7), $q \geq 2$ implies that

$$
\lambda_{T} \geq \sum_{i=1}^{k} \lambda_{T_{i}}+\sum_{i=l+1}^{k} \lambda_{T_{i}}
$$

If $l \leq k-1$ then, by induction,

$$
\sum_{i=l+1}^{k} \lambda_{T_{i}} \geq \sum_{i=l+1}^{k} \text { ord } T_{i} \geq 1,
$$

therefore (again by induction)

$$
\lambda_{T} \geq \sum_{i=1}^{k} \text { ord } T_{i}+1
$$

Since $l \leq k-1$,

$$
\operatorname{ord} T=\sum_{i=1}^{k} \operatorname{ord} T_{i}+1
$$

(since exactly one extra vertex is added by composition) and this proves the inductive step. In the remaining case, $l=k$, we have by induction 


$$
\lambda_{T}=\sum_{i=1}^{k} \lambda_{T_{i}} \geq \sum_{i=1}^{k} \operatorname{ord} T_{i}=T,
$$

because no vertices are added in the composition. The proof of the second part of the lemma follows.

Finally, to prove the last assertion, we let $T^{(0)}:=T_{\circ}$ and define by induction

$$
T^{(j+1)}:=T^{(j)}-\overbrace{T_{\circ}-T_{\circ}-\cdots-T_{\circ}}^{k-1 \text { times }}, \quad j=0,1, \ldots
$$

Since $a_{k, k} \neq 0$, we have $T^{(j+1)} \in \mathbb{T}$. It follows easily by induction that $\lambda_{T^{(j)}}=$ ord $T^{(j)}=j(k-1)+1, j \in \mathbb{Z}^{+}$. This completes the proof of the lemma.

The implication of the last part of the lemma is that in most cases the bound $\lambda_{T} \geq \operatorname{ord} T$ for $q \geq 2$ is the best possible. However, the main significance of Lemma 19 is in establishing that we may take $\eta=\min \{1, q-1\}>0$ whenever $q>1$. Needless to say, the larger the value of $\eta$, the more powerful are the results of $\S 4$.

The set $\mathbb{T}$ and the functions $\beta$ and $\gamma$ can be worked out explicitly in special cases and we have already seen this for the equation (5.1). Herewith we consider two further examples. Firstly, the equation

$$
y^{\prime}(t)=b y(t)+a_{2,2} y^{2}(t)+a_{1,0} y(q t), \quad y(0)=y_{0} .
$$

Asserting $a_{1,0}, a_{2,2} \neq 0$, we have

$$
T_{1}, T_{2} \in \mathrm{T} \quad \Rightarrow \quad T_{1}-T_{2},{\stackrel{1}{T_{1}} \in \mathrm{T}}^{2}
$$

and it is an easy matter to deduce that $\mathbb{T}=\mathbb{W}$, the set of all multiply rooted planar trees.

To gain intuition on $\beta$ and $\gamma$ we examine in Table 3 all members of $\mathbb{T}$ of order $\leq 4$, together with the corresponding values of $\gamma$. For brevity, the value of $\beta$ is not listed, but in all these instances it is consistent with the identity $\beta(T)=$ ord $T-\gamma(T)$.

Proposition 20. In the case of the equation (5.8), the value of $\gamma(T)$ for every $T \in \mathbb{T}$ equals the number of vertices of $T$ that possess children, whereas $\beta(T)=$ ord $T-\gamma(T)$.

Proof. Let $\varphi(T)$ denote the number of vertices of $T$ that possess children and let $\chi(T):=\beta(T)+\gamma(T)$. Hence

1. $\varphi\left(T_{\circ}\right)=0, \chi(T)=1$;

2. $T=T_{1}-T_{2} \rightarrow \varphi(T)=\varphi\left(T_{1}\right)+\varphi\left(T_{2}\right), \chi(T)=\chi\left(T_{1}\right)+\chi\left(T_{2}\right)$;

3.

$$
T=\rfloor^{T_{1}} \Rightarrow \varphi(T)=\varphi\left(T_{1}\right)+1, \chi(T)=\chi\left(T_{1}\right)+1,
$$

and we deduce that, whilst both functions share the same recurrence relation with $\gamma(T)$ and ord $T, \varphi$ is consistent with the initial value of $\gamma$, whereas $\chi\left(T_{\circ}\right)=\operatorname{ord} T_{\circ}$. 
Table 3. All $T \in \mathbb{T}$, ord $T \leq 4$, for equation (5.8)

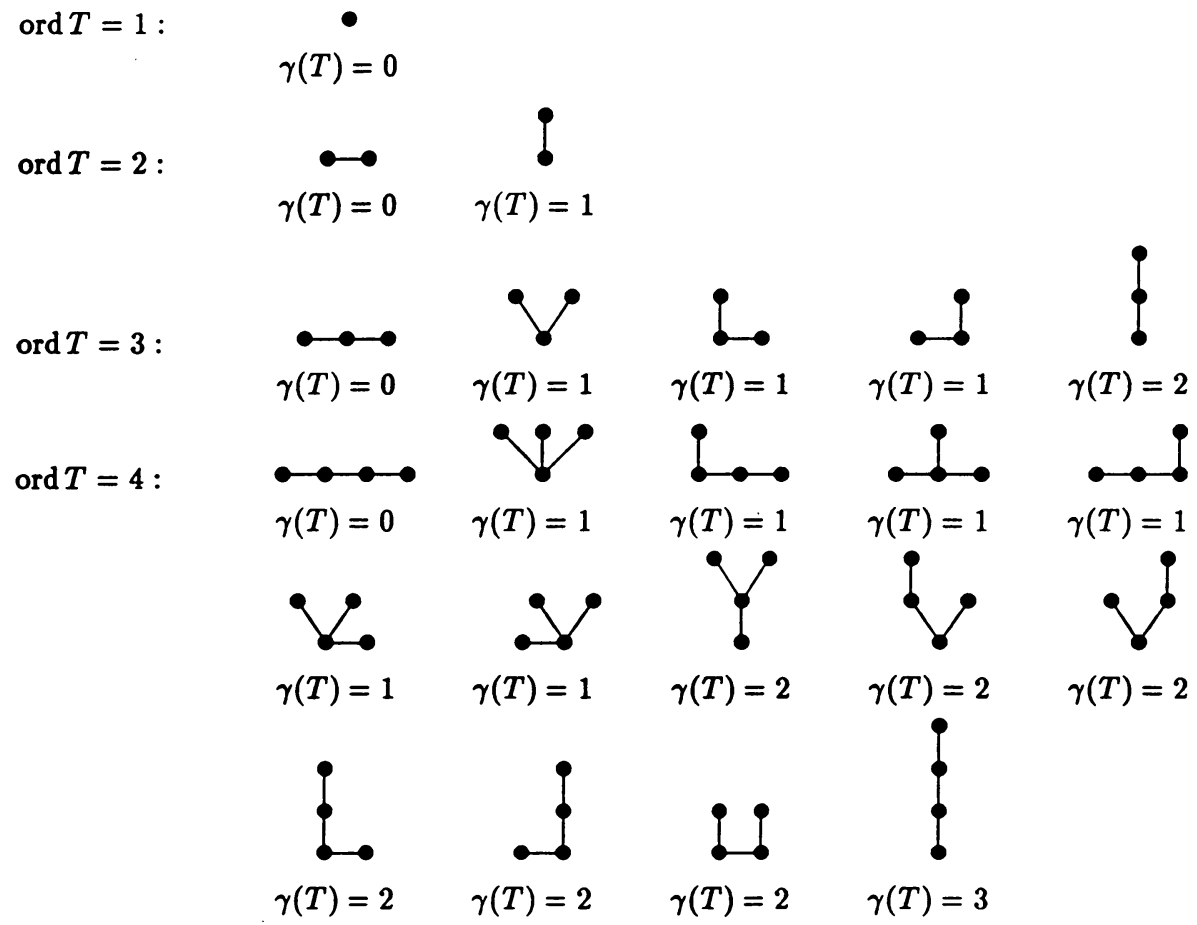

Our next-and last-example is the equation

$$
y^{\prime}(t)=b y(t)+a_{2,1} y(t) y(q t), \quad y(0)=y_{0} .
$$

We assume that $a_{2,1} \neq 0$.

Proposition 21. If $T \in \mathbb{T}$ for (5.9) then necessarily ord $T$ is odd.

Proof. Clearly, ord $T_{\circ}=1$, an odd number. We continue by induction. The only extant composition rule is

$$
T_{1}, T_{2} \in T \quad \Rightarrow \quad T_{1} \overbrace{}^{T_{2}} \in T .
$$

Therefore ord $T=$ ord $T_{1}+$ ord $T_{2}+1$, and, provided that ord $T_{1}$ and ord $T_{2}$ are odd, so is ord $T$.

Table 4 displays all members of $\mathbb{T}$ of order $\leq 7$.

Proposition 22. Let $T \in \mathbb{T}$, ord $T=2 n+1$. Then $\beta(T)=n+1, \gamma(T)=n$. Proof. Let us suppose that ord $T_{i}=2 k_{i}+1, k_{1}+k_{2}=n-1$ (recall that, by Proposition 21 , all $T \in \mathbb{T}$ have odd order). Then, by the composition rule (5.10) and induction on $n$,

$$
\begin{aligned}
& \beta(T)=\beta\left(T_{1}\right)+\beta\left(T_{2}\right)=\left(k_{1}+1\right)+\left(k_{2}+1\right)=n+1 ; \\
& \gamma(T)=\gamma\left(T_{1}\right)+\gamma\left(T_{2}\right)+1=k_{1}+k_{2}+1=n \text {. Q.E.D. }
\end{aligned}
$$


Table 4. All $T \in \mathbb{T}$ for the equation (5.9) with ord $T \leq 7$

$\operatorname{ord} T=1:$

$\operatorname{ord} T=3:$

$\operatorname{ord} T=5:$

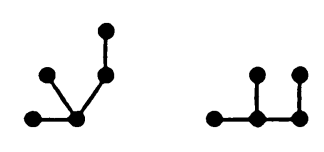

$\operatorname{ord} T=7:$
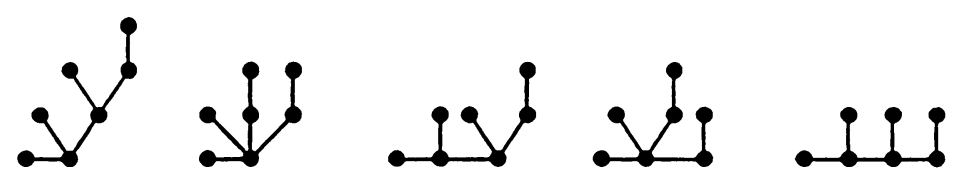

Proposition 23. Let $T \in \mathbb{T}$, ord $T=2 n+1$. Then there exists a polynomial $p_{T}$ with nonnegative integer coefficients such that $p_{T}(0)$ is a positive integer and $\alpha(T)=q^{n} p_{T}(q)$.

Proof. We recall that each $T \in \mathbb{T}$ is of odd order, and assume again that, for $n \geq 1, T$ is obtained from $T_{1}$ and $T_{2}$ as in (5.10). Therefore,

$$
\lambda_{T}=\lambda_{T_{1}}+q \lambda_{T_{2}}
$$

and we deduce that $\lambda_{T}=1+\mathscr{O}(q), q \rightarrow 0$. Moreover,

$$
\alpha(T)=\left(\lambda_{T}-1\right) \alpha\left(T_{1}\right) \alpha\left(T_{2}\right) .
$$

The statement of the proposition is true for $n=0$ and we continue by induction. Given $n \geq 1,(5.11)$ and (5.12) yield

$$
\alpha(T)=\left(\lambda_{T_{1}}+q \lambda_{T_{2}}\right) q^{k_{1}} p_{T_{1}}(q) q^{k_{2}} p_{T_{2}}(q)=\left(\lambda_{T_{1}}+q \lambda_{T_{2}}\right) q^{n-1} p_{T_{1}}(q) p_{T_{2}}(q) .
$$

Moreover,

$$
\lim _{q \rightarrow 0+} q^{-1}\left(\lambda_{T_{1}}+q \lambda_{T_{2}}-1\right)=\lim _{q \rightarrow 0+} q^{-1}((1+\mathscr{O}(q))+q(1+\mathscr{O}(q))-1)=\mathscr{O}(1),
$$

therefore $q^{-n} \alpha(T)=\mathscr{O}(1), q \rightarrow 0$, and

$$
p_{T}(q)=\left(\frac{\lambda_{T_{1}}-1}{q}+\lambda_{T_{2}}\right) p_{T_{1}}(q) p_{T_{2}}(q) .
$$

Finally, it is easy to demonstrate that $p_{T}(0) \in\{1,2,3, \ldots\}$. Since each $\lambda_{T}$ is a polynomial in $q$ with nonnegative integer coefficients and $\lambda_{T_{1}}=1+\mathscr{O}(1)$, it follows that

$$
\lim _{q \rightarrow 0+}\left(\frac{\lambda_{T_{1}}-1}{q}+\lambda_{T_{2}}\right)=\left.\frac{d \lambda_{T_{1}}}{d q}\right|_{q=0}+1
$$

is a natural number. Hence, and since $p_{T_{\circ}} \equiv 1$, an induction on (5.13) affirms that $p_{T}(0)$ is itself a natural number for all $T \in \mathbb{T}$. 
Substitution into (5.9), in tandem with the last two propositions, affirms that

$$
d_{T}=\frac{1}{p_{T}} d_{T_{\circ}}\left(\frac{d_{T_{\circ}} \omega}{q}\right)^{n},
$$

where ord $T=2 n+1$ and $\omega=a_{2,1} / b$. Moreover, $p_{T}(q) \geq 1$ for all $T \in \mathbb{T}$, and we deduce that

$$
\left|d_{T}\right| \leq\left|d_{T_{\circ}}\right|\left(\frac{\left|d_{T_{\circ}} \omega\right|}{q}\right)^{n}
$$

Let us denote by $\mathbb{D}_{n}$ all $T \in \mathbb{T}$ of order $2 n+1$. According to Proposition 21 , $\mathbb{T}=\bigcup_{n \in \mathbb{Z}^{+}} \mathbb{D}_{n}$. We let $\widetilde{M}_{n}$ stand for the number of elements in $\mathbb{D}_{n}$. Then, by (5.14),

$$
d^{*} \leq\left|d_{T_{\circ}}\right| \widetilde{M}\left(\left|d_{T_{\circ}} \omega\right| / q\right),
$$

where

$$
\widetilde{M}(x):=\sum_{n=0}^{\infty} \widetilde{M}_{n} x^{n} .
$$

Proposition 24. The Taylor expansion of $\widetilde{M}$ converges for all $|x|<\frac{1}{4}$ and

$$
\widetilde{M}(x)=\frac{1-\sqrt{1-4 x}}{2 x}=\sum_{n=0}^{\infty} \frac{(2 n) !}{n !(n+1) !} x^{n} .
$$

Therefore, $\widetilde{M}_{n}=(2 n) ! /(n !(n+1) !)$.

Proof. Suppose that $T \in \mathbb{D}_{n}, n \geq 1$ has been obtained from (5.10). Hence there exists $k \in\{0,1, \ldots, n-1\}$ such that $T_{1} \in \mathbb{D}_{k}, T_{2} \in \mathbb{D}_{n-k-1}$. It follows that

$$
\widetilde{M}_{n}=\sum_{k=0}^{n-1} \widetilde{M}_{k} \widetilde{M}_{n-k-1}, \quad n=1,2, \ldots
$$

Multiplying the last expression by $x^{n}$, summing up for $n=1,2, \ldots$ and adding $\widetilde{M}_{0}=1$ readily yields

$$
\widetilde{M}(x)=1+\sum_{n=1}^{\infty} \sum_{k=0}^{n-1} \widetilde{M}_{k} \widetilde{M}_{n-k-1} x^{n}=1+x \widetilde{M}^{2}(x) .
$$

It follows that $\widetilde{M}$ is a solution of the quadratic equation $x t^{2}-t+1=0$. Since $\widetilde{M}(0)=1$, it follows that $M(x)=(1-\sqrt{1-4 x}) /(2 x)$, with the radius of convergence $\frac{1}{4}$. The remainder of the lemma is a consequence of a straightforward Taylor expansion of the square root function.

Theorem 25. For every $\left|d_{T_{\circ}}\right|<q /(4|\omega|)$ the Dirichlet expansion of the equation (5.9) converges in an interval of the form $\left[0, t^{*}\right)$, where $t^{*}=\infty$ if $\operatorname{Re} b \leq 0$.

Proof. A straightforward consequence of our analysis and of $\S 3$.

As before, we can deduce from the last theorem that $\operatorname{Re} b<0$ implies asymptotic stability, whereas $\operatorname{Re} b=0, b \neq 0$, implies almost periodicity. Likewise, rephrasing in terms of $y(0)$ rather than $d_{T_{\circ}}$, we can deduce asymptotic stability or almost periodicity, as the case might be, subject to $|y(0)|$ being sufficiently small. 
The last example does not fit into the pattern of Lemma 18 and, indeed, it displays different behaviour altogether-Dirichlet series converge regardless of the value of $q>0$, as long, of course, as $\left|d_{T_{\circ}}\right|$ is sufficiently small.

The radius of convergence of $\widetilde{M}$ (after an obvious change of variables) is more generous than of the function $M$ from $\S 4$. This should come as no surprise, since $M$ has been derived by counting all members of $\mathbb{W}$. In the case of (5.9) all the $a_{k, l} s$, save for one, vanish and $\mathbb{T}$ forms a small subset of $\mathbb{W}$.

\section{A RATIONAL EQUATION}

A natural step in generalizing (5.4) consists of replacing a bivariate polynomial in $y(t)$ and $y(q t)$ by a bivariate rational function. In the present section we present a preliminary result of that kind. For the sake of clarity we restrict our analysis to the simple equation

$$
y^{\prime}(t)=\frac{b y(t)}{1+a y(q t)}, \quad y(0)=y_{0},
$$

where $q>0$ and $a, b \neq 0$. Letting again

$$
y(t)=\sum_{T \in \mathbb{T}} d_{T} e^{b \lambda_{T} t}, \quad t \geq 0,
$$

we derive upon substitution into (6.1) the equation

$$
\begin{aligned}
\sum_{T \in \mathbb{T}} & \left(1-\lambda_{T}\right) d_{T} \exp \left\{b \lambda_{T} t\right\} \\
& =a \sum_{T_{1}, T_{2} \in \mathbb{T}} \lambda_{T_{1}} d_{T_{1}} d_{T_{2}} \exp \left\{b\left(\lambda_{T_{1}}+q \lambda_{T_{2}}\right)\right\} .
\end{aligned}
$$

It is easy to deduce that the composition rules for the equation (5.9) are valid, hence the set $\mathbb{T}$ is the same as in the latter part of $\S 5$ and Table 4 . However, the recurrence for $d_{T}$ is different and it reads

$$
d_{T}=\frac{a \lambda_{T_{1}}}{1-\lambda_{T}} d_{T_{1}} d_{T_{2}}
$$

$T$ having been derived from $T_{1}$ and $T_{2}$ by (5.10).

Bearing in mind Proposition 21, we can prove, by revisiting the technique of the previous section, that

$$
d_{T}=\frac{d_{T_{\circ}}}{r_{T}}\left(\frac{d_{T_{\circ}} a}{q}\right)^{n}, \quad \text { ord } T=2 n+1,
$$

where

$$
r_{T}=r_{T}(q):=-\frac{\lambda_{T_{2}}+q^{-1}\left(\lambda_{T_{1}}-1\right)}{\lambda_{T_{1}}} r_{T_{1}}(q) r_{T_{2}}(q) .
$$

Since $\lambda_{T}=1+\mathscr{O}(q), q \rightarrow 0$, it follows that $r_{T}$ is a rational function in $q$ and that $\left|r_{T}\right|$ has nonnegative coefficients. Table 5 displays the functions $p_{T}$ (from $\S 5)$ and $r_{T}$ for ord $T \leq 7$.

An examination of Table 5 is suggestive of the assertion that $p_{T}$ is the numerator of $r_{T}$. This can be easily proved, dividing (5.13) by (6.4),

$$
\left(\frac{p_{T}}{r_{T}}\right)=-\lambda_{T_{1}}\left(\frac{p_{T_{1}}}{r_{T_{1}}}\right)\left(\frac{p_{T_{2}}}{r_{T_{2}}}\right) .
$$


Table 5. Functions $p_{T}$ and $r_{T}$ for ord $T \leq 7$

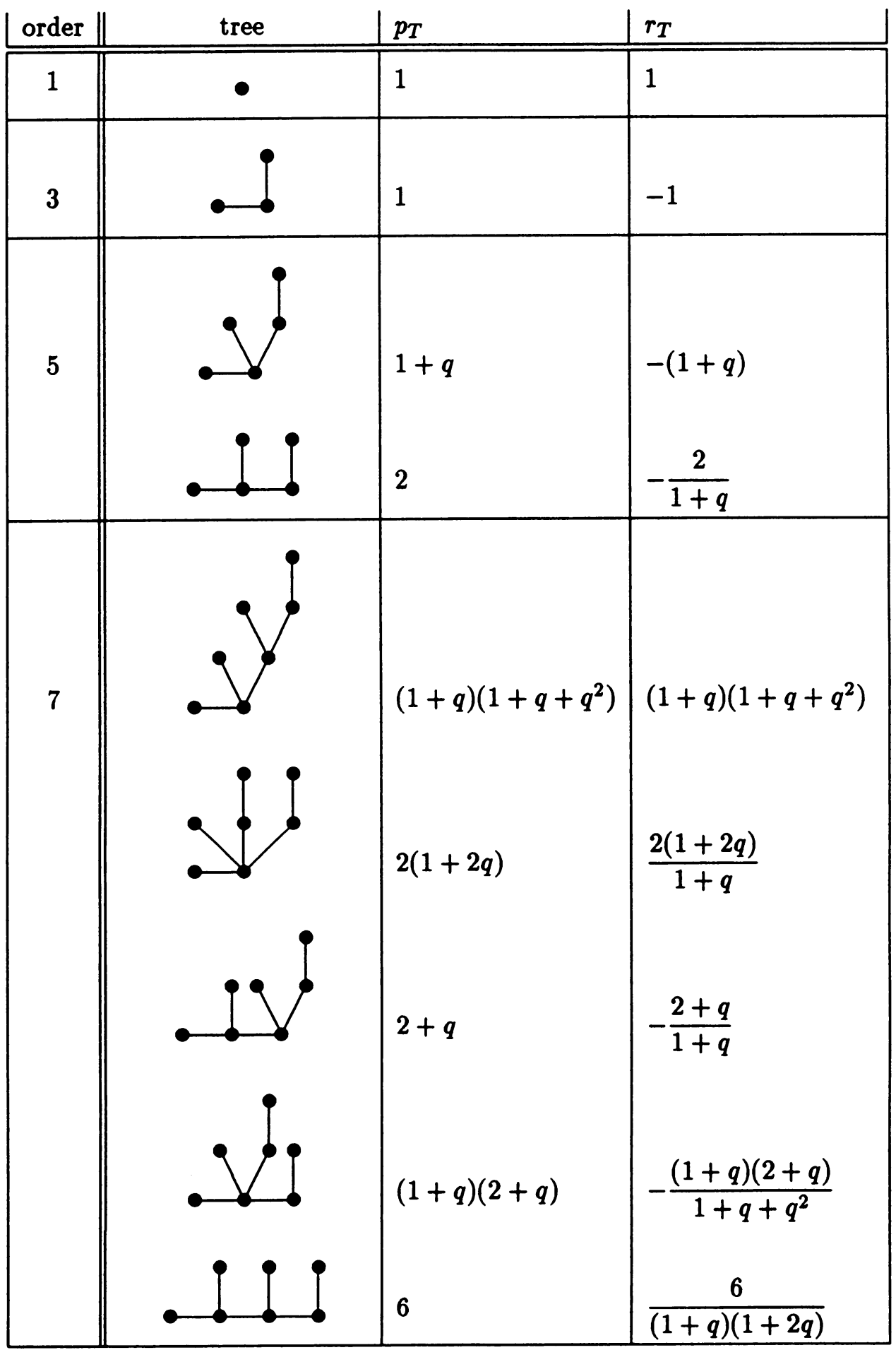


Since $p_{T_{\circ}} / r_{T_{\circ}}=1$, it follows by induction that $p_{T} / r_{T}$ is a polynomial, consequently $p_{T}$ is indeed a numerator of $r_{T}$. We conclude that, since the denominator of $r_{T}$ has nonnegative coefficients, necessarily $\left|r_{T}\right| \leq p_{T}$. Therefore, if the Dirichlet series for (6.1) converges, then so does the Dirichlet series for (5.9) (with $a$ replaced by $\omega$ ). This, however, is of limited significance, since we already possess in Theorem 25 a convergence condition for Dirichlet expansions to $(5.9)$.

Let us suppose that $q>1$ and set

Therefore,

$$
F(x, y):=\frac{x}{y+\frac{x-1}{q}} .
$$

$$
\left|r_{T}^{-1}\right|=F\left(\lambda_{T_{1}}, \lambda_{T_{2}}\right)\left|r_{T_{1}}^{-1}\right|\left|r_{T_{2}}^{-1}\right|,
$$

provided that $T$ has been composed from $T_{1}$ and $T_{2}$. It is straightforward to verify that $q>1$ implies that $\partial F / \partial x>0, \partial F / \partial y<0$ for all $x, y>1$.

Proposition 26. Provided that $q>1$, every $T \in \mathbb{D}_{n}$ obeys the inequality

$$
1+n q \leq \lambda_{T} \leq 1+q+\cdots+q^{n} .
$$

Moreover, there exist $U_{n}, V_{n} \in \mathbb{D}_{n}$ such that

$$
\lambda_{U_{n}}=1+n q, \quad \lambda_{V_{n}}=1+q+\cdots+q^{n} .
$$

Proof. We use induction on $n$. (6.6) is certainly true for $n=0$. Otherwise there exists $k \in\{0,1, \ldots, n-1\}$ such that

$$
\begin{aligned}
\lambda_{T} & =\lambda_{T_{1}}+q \lambda_{T_{2}} \quad T_{1} \in \mathbb{D}_{k}, T_{2} \in \mathbb{D}_{n-k-1} \\
& \leq \sum_{j=0}^{k} q^{j}+q \sum_{j=0}^{n-k-1} q^{j}=\sum_{j=0}^{n} q^{j}-(q-1) \sum_{j=1}^{n-k} q^{j} .
\end{aligned}
$$

Since $q>1$, the upper bound is true. Lower bound can be proved in an identical fashion.

To prove that both bounds are attainable, we specify $U_{n}$ and $V_{n}$ explicitly,

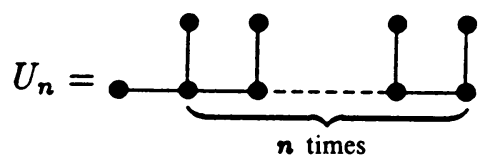

and

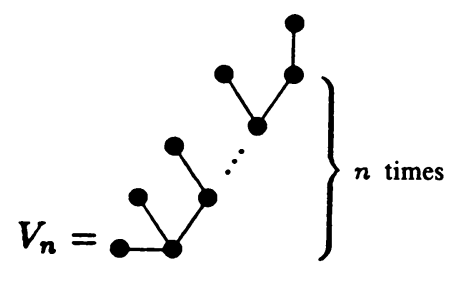

It is trivial to prove by induction that $U_{n}, V_{n} \in \mathbb{D}_{n}$ and that they obey the lower and the upper bound, respectively, since $U_{n}$ is composed from $U_{n-1}$ and $T_{0}$, whilst $V_{n}$ is composed from $T_{\circ}$ and $V_{n-1}$.

Corollary. For every $n \geq 1$ and $k \in\{0,1, \ldots, n-1\}$ it is true that

$$
F\left(\lambda_{T_{1}}, \lambda_{T_{2}}\right) \leq F\left(\lambda_{V_{k}}, \lambda_{U_{n-k-1}}\right), \quad T_{1} \in \mathbb{D}_{k}, T_{2} \in \mathbb{D}_{n-k-1} .
$$

Proof. Follows at once from (6.6), since $F$ increases monotonically in its first argument and decreases in the second. 
Let

$$
\begin{aligned}
h_{k} & :=F\left(\lambda_{V_{k}}, \lambda_{U_{n-k-1}}\right) \\
& =\frac{q^{k+1}-1}{q^{k}+(n-k-1) q^{2}-(n-k-2) q-2}, \quad k=0,1, \ldots, n-1 .
\end{aligned}
$$

It is an elementary exercise to prove that $h_{k+1}>h_{k}, k=0,1, \ldots, n-2$. Consequently,

$$
h_{k} \leq h_{n-1}=\frac{q^{n}-1}{q^{n-1}+q-2} \leq q, \quad k=0,1, \ldots, n-1 .
$$

It follows from (6.7) that $F\left(\lambda_{T_{1}}, \lambda_{T_{2}}\right) \leq q$ and we deduce from (6.5) that

$$
\left|r_{T}^{-1}\right| \leq q\left|r_{T_{1}}^{-1}\right| r_{T_{2}}^{-1} \mid
$$

Let us assume that $\left|r_{T_{j}}^{-1}\right| \leq q^{\psi\left(T_{j}\right)}, j=1,2$. Then, according to the inequality (6.8), $\left|r_{T}^{-1}\right| \leq q^{\psi\left(T_{1}\right)+\psi\left(T_{2}\right)+1}$. We conclude that $\left|r_{T}^{-1}\right| \leq q^{\psi(T)}$, where $\psi\left(T_{\circ}\right)=0$ and $\psi(T)=\psi\left(T_{1}\right)+\psi\left(T_{2}\right)+1$. Thus, $\psi$ obeys exactly the same recurrence and initial condition as $\gamma$ and, by Proposition 22,

$$
\left|r_{T}^{-1}\right| \leq q^{n}, \quad T \in \mathbb{D}_{n}
$$

Theorem 27. Let $q>1$. Then, for every $\left|d_{T_{\circ}}\right|<1 /(4|a|)$, the Dirichlet expansion of the equation (6.1) converges in an interval of the form $\left[0, t^{*}\right)$, where $t^{*}=\infty$ if $\operatorname{Re} b \leq 0$.

Proof. Similar to the proof of Theorem 25, except that, by virtue of (6.9), (5.15) need be replaced by

$$
\left|d_{T}\right| \leq\left|d_{T_{\circ}}\right|\left|d_{T_{\circ}} a\right|^{n}, \quad n \in \mathbb{D}_{n} .
$$

As before, the existence of a Dirichlet expansion, in tandem with the material of $\S 3$, go a long way towards explaining the asymptotic behaviour of $y$ as $t \gg 0$ in the case $\operatorname{Re} b \leq 0$.

The remaining case, $q \in(0,1)$, is considerably easier.

Theorem 28. Provided that $q \in(0,1)$, the Dirichlet expansion of the equation (6.1) converges in an interval of the form $\left[0, t^{*}\right)$, where $t^{*}=\infty$ if $\operatorname{Re} b \leq 0$, provided that $\left|d_{T_{\circ}}\right|<q /(4|a|)$.

Proof. Given $T_{1}, T_{2} \in \mathbb{T}$, set

$$
G(t):=\frac{\lambda_{T_{1}}}{\lambda_{T_{2}}+t^{-1}\left(\lambda_{T_{1}}-1\right)} .
$$

Since $\lambda_{T_{1}}, \lambda_{T_{2}} \geq 1, G$ is monotone in $t$ and 


$$
0<G(t) \leq G(1)=\frac{\lambda_{T_{1}}}{\lambda_{T_{1}}+\lambda_{T_{2}}-1} \leq 1 .
$$

Therefore we deduce from (6.4) that $\left|r_{T}^{-1}\right| \leq\left|r_{T_{1}}^{-1}\right|\left|r_{T_{2}}^{-1}\right|$. The remainder of the proof follows along identical lines to that of Theorems 25 and 27.

\section{REFERENCES}

1. C. Berge, The theory of graphs and its applications, Wiley, New York, 1962.

2. F. Harary, Graph theory, Addison-Wesley, Reading, Mass., 1969.

3. G. H. Hardy and M. Riesz, The general theory of Dirichlet's series, Cambridge Univ. Press, Cambridge, 1915.

4. A. Iserles, On the generalized pantograph functional-differential equation, European J. Appl. Math. 4 (1992), 1-38.

5. T. Kato and J. B. McLeod, The functional-differential equation $y^{\prime}(x)=a y(\lambda x)+b y(x)$, Bull. Amer. Math. Soc. 77 (1971), 891-937.

6. T. W. Körner, Fourier analysis, Cambridge Univ. Press, Cambridge, 1988.

Department of Applied Mathematics and Theoretical Physics, University of CamBRIDGE, CAMBRIDGE, ENGLAND

E-mail address: A. Iserles@amtp.cam.ac.uk 